This item was submitted to Loughborough's Research Repository by the author.

Items in Figshare are protected by copyright, with all rights reserved, unless otherwise indicated.

\title{
3D Heterogeneous stiffness reconstruction using MRI and the virtual fields method
}

PLEASE CITE THE PUBLISHED VERSION

http://dx.doi.org/10.1007/s11340-008-9128-2

PUBLISHER

Springer ( ) Society for Experimental Mechanics

VERSION

AM (Accepted Manuscript)

LICENCE

CC BY-NC-ND 4.0

REPOSITORY RECORD

Avril, S., Fabrice Pierron, Jonathan M. Huntley, and D.D. Steele. 2019. "3D Heterogeneous Stiffness

Reconstruction Using MRI and the Virtual Fields Method”. figshare. https://hdl.handle.net/2134/13247. 
This item was submitted to Loughborough's Institutional Repository (https://dspace.lboro.ac.uk/) by the author and is made available under the following Creative Commons Licence conditions.

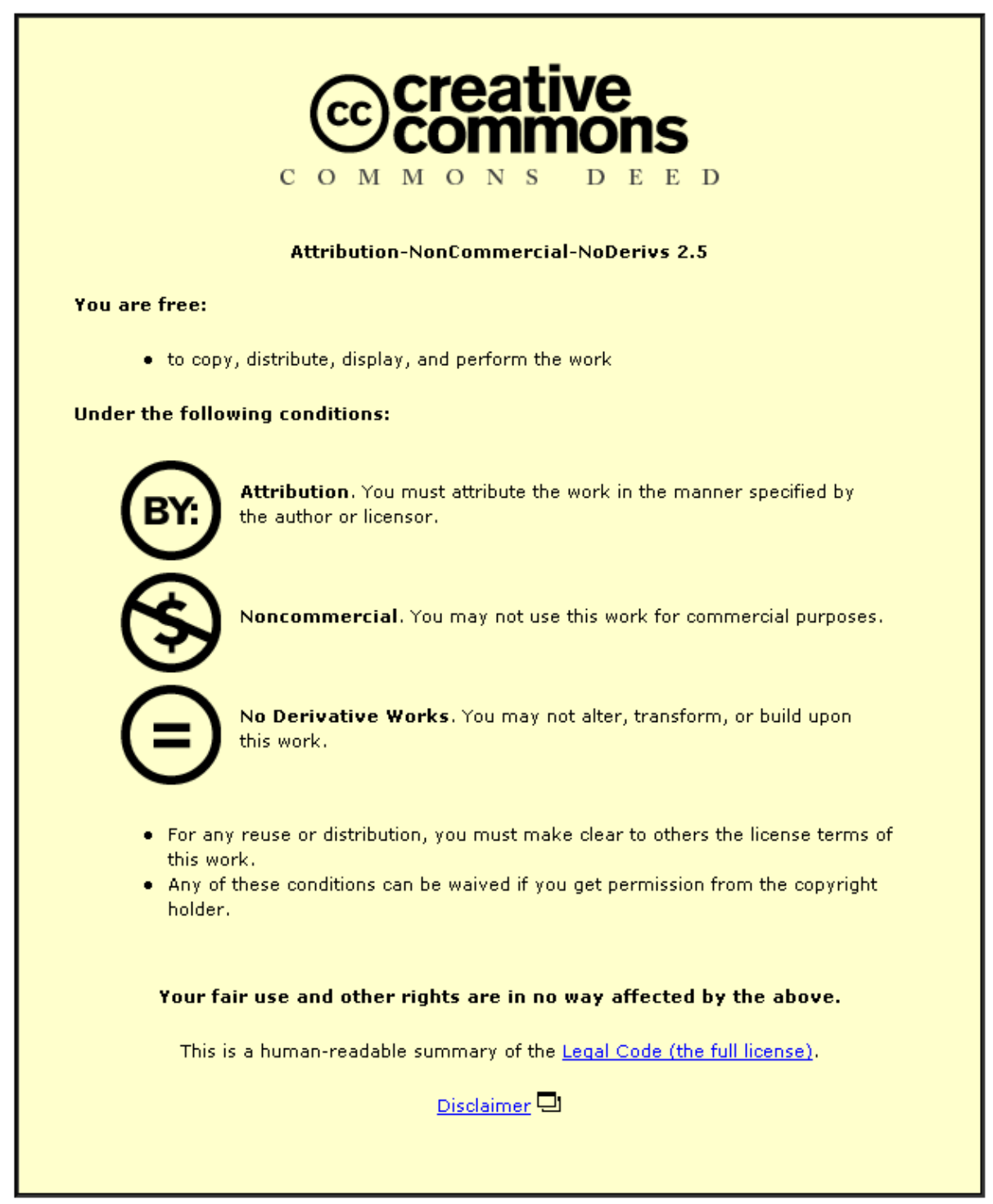

For the full text of this licence, please go to: http://creativecommons.org/licenses/by-nc-nd/2.5/ 


\title{
3D heterogeneous stiffness identification using MRI and the virtual fields method.
}

\author{
S. Avril ${ }^{1,2}$, J.M. Huntley ${ }^{2}$, F. Pierron ${ }^{1}$, D.D. Steele $^{3}$
}

1 LMPF-ENSAM, rue Saint Dominique, BP 508, 51006 Châlons en Champagne, France

2 Wolfson School of Mechanical and Manufacturing Engineering, Loughborough University, Loughborough, Leicestershire LE11 3TU, UK

3 Departments of Radiology and Biomedical Engineering, University of Michigan Medical Center, 3315 Kresge III, Ann Arbor, MI 48109-0553, USA

\begin{abstract}
The first extension of the virtual fields method to the identification of heterogeneous stiffness properties from 3D bulk full-field displacement data is presented in this paper. Data are provided by Magnetic Resonance Imaging (MRI). Two main issues are addressed: 1 . the identification of the stiffness ratio between two different media in a heterogeneous solid; 2 . the identification of stiffness heterogeneities buried in a heterogeneous solid. The approach is based on a finite element discretization of the equilibrium equations. It is tested on experimental full-field data obtained on a phantom with the stimulated echo MRI technique. The application deals with the characterization of a stiff spherical inclusion buried within a lower modulus material. Results concerning the ratio between the modulus of the inclusion and the modulus of the surrounding material are in close agreement with the reference. Results concerning the reconstructed modulus distribution across the whole investigated volume are also quite promising. The inclusion location and the ratio of the average moduli are in agreement with the reference. However, the resulting modulus distribution is highly variable. This is explained by the fact that the approach relies on a second order differentiation of the data, which tends to amplify noise. It is eventually proved that noise can be removed by using filtering algorithms.
\end{abstract}

Key words: Virtual Fields Method; MRI; Elastography; 3D full-field measurements, Identification. 


\section{Introduction}

In medicine, the accurate characterization of stiffness properties inside the human body is essential in order to detect pathological tissues and diagnose disease [Ophir et al., 2001, Manduca et al., 2001]. This entails addressing a challenging issue within the field of solid mechanics: the non intrusive identification of mechanical properties inside a deformable body. This issue can be addressed in many different ways, all involving the definition of a so-called inverse problem [Bui, 1994, Tarantola, 2005, Bonnet and Constantinescu, 2005]. Thanks to ultrasound [Ophir et al., 2001, Fehrenbach et al., 2006] and magnetic resonance imaging (MRI) [MacRobbie et al., 2003,Steele et al., 2000], it is possible to measure displacement fields in many human tissues. The approaches used to process these full-field data depend on the way the solid is deformed. Two different ways exist:

- static experiments: the tissue is compressed quasi-statically [Barbone and Gokhale, 2004,Skovoroda et al., 1995, Skovoroda et al., 1999, Fehrenbach et al., 2006],

- dynamic experiments: a time harmonic excitation made on the boundary creates a time harmonic shear wave in the tissue [McLaughlin and Yoon, 2004, Weaver et al., 2005, Manduca et al., 2001, Romano et al., 2001, Liew and Pinsky, 2005, Doyley et al., 2005, Park and Maniatty, 2006].

Many applications use dynamic experiments. The advantage over quasi-static experiments is that a direct relationship between the measured strains and the unknown moduli can be derived, provided that only shear waves are present in the tissues [McLaughlin and Yoon, 2004,Park and Maniatty, 2006]. Such a direct relationship is usually not available in static experiments. A map of the quasi-static strain distribution in tissue reveals not only information about tissue shear modulus distributions, but also about tissue connectivity (interfaces between tissue components) and other geometrical considerations at the boundary [Ophir et al., 2001]. Thus, an inverse problem has to be solved for identifying only tissue shear modulus distributions independently of the boundary conditions [Doyley et al., 2005].

The inversion to obtain stiffness distributions using quasi-static experiments has been solved in two ways in the literature. The first one is a direct method which was developed by Skovodora et al. [Skovoroda et al., 1995, Skovoroda et al., 1999] and the solvability was investigated by [Barbone and Gokhale, 2004]. The principle of this method is to cancel the curl of the equilibrium equations. This yields a hyperbolic partial differential equation (PDE) with the shear modulus distribution as the unknown [Skovoroda et al., 1995]. This approach presents some drawbacks, the main one being that it is required to differentiate at least two times the noise-corrupted displacement fields, yielding artifacts in the results [Steele et al., 2000]. Alternative formulations of this approach have been proposed by [Barbone and Oberai, 2007] but they require computing of the gradients of the hydrostatic pressure. This operation is highly unstable because gradients of the hydrostatic pressure are very small in nearly incompressible soft tissues, which means that they are experimentally negligible with respect to the measurement uncertainty, as this will be shown in this study. 
The second method of inversion is based on model updating [Liew and Pinsky, 2005, Doyley et al., 2005, Fehrenbach et al., 2006]. This approach tends to be computationally expensive since the forward problem has to be solved repeatedly. Even if the use of an adjoint approach significantly reduces the computation time, it remains a computationally-expensive method for processing 3D full-field measurements. Thus, 3D applications of model updating are scarce [Doyley et al., 2005].

In view of all the previous disadvantages, an alternative approach is proposed in this paper. It is based on a finite element discretization of the governing equations, like the approaches developed in 2D by [Claire et al., 2004] or [Park and Maniatty, 2006]. The idea here is to devise relevant combinations of the discretized equations by using the principle of the virtual fields method [Grédiac, 1989, Grédiac et al., 2006]. Until now, the virtual fields method has been an approach dedicated to engineering applications dealing with the identification of constitutive parameters of thin plates from 2D surface full-field kinematic measurements. Since the development of the virtual fields method, the choice of the virtual fields has been optimized [Avril et al., 2004], making possible the "direct" identification of stiffness parameters from full-field measurements [Grédiac et al., 2006]. As no iterative solution of the forward problem is required, this approach seems well suited for processing large amounts of data, and hence to 3D bulk displacement fields.

This paper presents the first extension of the virtual fields method to 3D bulk full-field displacement data obtained using the stimulated echo MRI technique [Steele et al., 2000]. The purpose is to determine 3D modulus distributions in solids that are assumed to behave in their linear elastic range. A promising experimental validation is shown for a cubic specimen containing a spherical inclusion four times stiffer than the surrounding material.

\section{Principle}

\subsection{General background}

\subsubsection{Definition of the inverse problem}

Let us consider a solid of any shape (Fig. 5.1) whose volume in the undeformed configuration is denoted $\Omega$. The mechanical behavior of this solid is assumed as linear, elastic and isotropic. In general, soft tissues in the human body are anisotropic, viscoelastic and nonlinear. However, it is usually assumed in elastography that they behave as linear, elastic, isotropic materials in order to simplify the analysis [Ophir et al., 2001, Manduca et al., 2001]. This is a linearized approximation but the distribution of "secant" moduli across soft tissues subjected to a given loading is of great interest for physicians to detect pathologies and should be regarded as a challenging target to reach. Therefore, this justifies the assumption of linear elasticity and isotropy used throughout this paper.

In static neglecting body forces and under the assumption of small perturbations, three sets of equations can be derived: 
- equilibrium equations:

$$
\left\{\begin{array}{l}
\operatorname{div} \boldsymbol{\sigma}=0 \text { in } \Omega \\
\boldsymbol{\sigma} \cdot \boldsymbol{n}=\overline{\boldsymbol{T}} \text { on } S_{f}
\end{array}\right.
$$

- kinematic compatibility equations

$$
\begin{cases}\varepsilon=\frac{1}{2}\left(\nabla \boldsymbol{u}+\nabla^{t} \boldsymbol{u}\right) & \text { in } \Omega \\ \boldsymbol{u}=\overline{\boldsymbol{u}} & \text { on } S_{u}\end{cases}
$$

- constitutive equations

$$
\boldsymbol{\sigma}=\boldsymbol{A} \varepsilon \text { in } \Omega
$$

where $\boldsymbol{u}$ denotes the displacement vector undergone by the solid due to external loading, $\boldsymbol{\varepsilon}$ the infinitesimal strain tensor, $\boldsymbol{\sigma}$ the Cauchy stress tensor, $\boldsymbol{A}$ the Hooke tensor linking the strains and stresses and $\boldsymbol{n}$ the outward unit normal vector. Overlined quantities are prescribed as static $(\overline{\boldsymbol{T}})$ or kinematic $(\overline{\boldsymbol{u}})$ boundary conditions. Let $S_{u}$ be the surface over which kinematic boundary conditions are prescribed and $S_{f}$ the surface over which static boundary conditions are applied (either external forces or nothing as in Fig. 5.1). The surfaces $S_{u}$ and $S_{f}$ are such that $S_{u} \cap S_{f}=\emptyset$ and $S_{u} \cup S_{f}=\partial \Omega$, so as to support a well-posed set of boundary conditions. $\partial \Omega$ denotes the complete surface enclosing $\Omega$.

If the elastic properties are assumed to be isotropic, the components of $\boldsymbol{A}$ may be described in terms of two independent scalar moduli: Young's modulus $E$ and Poisson's ratio $\nu$. Poisson's ratio is assumed to be constant across the volume of the solid whereas Young's modulus is allowed to be spatially varying (heterogeneous material).

Using the displacement field measurements available across the volume of the solid, the purpose of this study is both:

- to identify the ratio between the Young's modulus of different regions in a solid,

- to identify the quantitative modulus distribution in a solid.

\subsubsection{Approximation of displacement fields using a basis of piecewise trilinear functions}

Let us consider a parallelepiped included in the volume of the solid and across which displacement field measurements are available (i.e. the domain scanned by the measurement system). The study will focus on the distribution of Young's modulus across this parallelepiped.

For solving this inverse problem, the volume has to be discretized. The parallelepiped is partitioned into $N_{e}$ brick-shaped finite elements. Then, the displacement fields across the whole parallelepiped are approximated using the so-called Galerkin approximation [Zienkiewicz, 1977]. It involves replacing the displacement fields by the following series:

$$
\mathbf{u}(x, y, z) \approx \sum_{i=1}^{3 N_{n}} a_{i} \Phi_{i}(x, y, z)=<\Phi(x, y, z)>\{a\}
$$


where the $a_{i}$ 's denote the nodal degrees of freedom (DoF), $N_{n}$ the total number of nodes, and the $\Phi_{i}$ 's denote basis functions. All the nodal DoF values across the parallelepiped are brought together as a column vector denoted $\{a\}$. All the basis functions values are brought together as a row vector denoted $<\Phi>$.

The basis functions $\Phi_{i}(x, y, z)$ (where $x, y$ and $z$ are the usual cartesian coordinates) are equal to one at precisely one node of the mesh, and precisely zero at all other nodes. Such basis functions are built up in a piece-wise manner over the brick-shaped finite elements by combining trilinear shape functions which are only defined within a particular element [Zienkiewicz, 1977].

The components of vector $\{a\}$ are then identified by a least-squares regression. Indeed, the displacements are measured at a finite number of locations (voxel centers). Let $\{u\}$ denote the column vector containing the three components of all the displacement values measured at all the voxels in the volume scanned by the measurement technique. Afterwards, the displacement field is reconstructed everywhere across the volume of interest using a linear combination of basis functions. Let $[\Phi]$ denote the matrix containing all the values taken by the basis functions at all the voxel centres. The coefficients of the linear combination have to be determined so that the displacement reconstructed at the voxel centres matches at best the measured displacement. Therefore, the $\{a\}$ vector, which is the solution of the least squares regression problem, can be written:

$$
\{a\}=\left[[\Phi]^{t}[\Phi]\right]^{-1}[\Phi]^{t}\{u\}
$$

Accordingly, the size of the finite element mesh must be determined as a function of the spatial resolution of displacement field measurements. The number of rows in matrix $[\Phi]$ must be larger than the number of columns to ensure the existence of a unique solution to the least squares regression problem. Thus one may use as many DoFs as there are measured displacement values. It is however advisable to use significantly fewer DoFs when measurements are noisy for filtering purposes.

\subsubsection{Construction of the stiffness matrix}

The Young's modulus is assumed to be constant inside each brick-shaped finite element. Hence, the inverse problem is reduced to the determination of a vector, denoted $\{E\}$, whose components $E_{k}$ are the unknown Young's moduli of all the finite elements.

The stiffness matrix of a single brick finite element $e_{k}$ can be written:

$$
\left[\mathcal{K}_{k}\right]=E_{k} \int_{e_{k}}[\nabla \Phi][C][\nabla \Phi] \mathrm{d} V
$$

where $[\nabla \Phi]$ is a matrix featuring the gradient of the basis functions of the $\langle\Phi\rangle$ row vector. $[C]$ is a matrix that only depends on Poisson's ratio [Zienkiewicz, 1977]. Indeed, for an isotropic material, Young's modulus may be factored out of the Hooke matrix yielding an alternate form of Hooke's law: 


$$
\left\{\begin{array}{l}
\sigma_{x x} \\
\sigma_{y y} \\
\sigma_{z z} \\
\sigma_{x y} \\
\sigma_{x z} \\
\sigma_{y z}
\end{array}\right\}=E \frac{1-\nu}{(1+\nu)(1-2 \nu)}\left[\begin{array}{cccccc}
1 & \frac{\nu}{1-\nu} & \frac{\nu}{1-\nu} & 0 & 0 & 0 \\
\frac{\nu}{1-\nu} & 1 & \frac{\nu}{1-\nu} & 0 & 0 & 0 \\
\frac{\nu}{1-\nu} & \frac{\nu}{1-\nu} & 1 & 0 & 0 & 0 \\
0 & 0 & 0 & \frac{1-2 \nu}{2(1-\nu)} & 0 & 0 \\
0 & 0 & 0 & 0 & \frac{1-2 \nu}{2(1-\nu)} & 0 \\
0 & 0 & 0 & 0 & 0 & \frac{1-2 \nu}{2(1-\nu)}
\end{array}\right]\left\{\begin{array}{l}
\varepsilon_{x x} \\
\varepsilon_{y y} \\
\varepsilon_{z z} \\
\varepsilon_{x y} \\
\varepsilon_{x z} \\
\varepsilon_{y z}
\end{array}\right\}
$$

The global stiffness matrix of the whole parallelepiped, denoted $[\mathcal{K}]$, is constructed by assembling all the elementary stiffness matrices:

$$
[\mathcal{K}]=\sum_{k=1}^{N_{e}} E_{k}\left[\mathcal{K}_{k}\right]
$$

Let us now write the equilibrium equations of the whole parallelepiped [Zienkiewicz, 1977]. Knowing the vector of nodal forces, denoted $\{b\}$, the following equation must be verified by the $E_{k}$ :

$$
[\mathcal{K}]\{a\}=\sum_{k=1}^{N_{e}} E_{k}\left[\mathcal{K}_{k}\right]\{a\}=\{b\}
$$

where the $\{a\}$ vector of nodal DoFs is the one derived from the least squares regression of experimentally measured displacements according to Eq. 2.5.

\subsubsection{Elimination of unknown reaction forces}

Nodal forces in a finite element may be separated into internal body forces and external forces [Zienkiewicz, 1977]. Here, the body forces are neglected. Therefore, in Eq. 2.9, all the nodal forces cancel out except at the nodes located over the boundary of the parallelepiped where the reaction forces are unknown. Thus, vector $\{b\}$ can be split up in two sub-vectors, one containing all the known components, which are all zeros, and one containing the unknown components, denoted $\{F\}$. The number of unknown components is denoted $N_{f}$. Those unknown nodal forces are removed from the equations. After this operation, the number of remaining equations is $N_{e}-N_{f}$. Similarly, all vectors are split up into two sub-vectors, and all matrices are split up into four sub-matrices, such as:

$$
\{a\}=\left\{\frac{\{U\}}{\{V\}}\right\} \quad\{b\}=\left\{\frac{\{0\}}{\{F\}}\right\} \quad[\mathcal{K}]=\left[\frac{[K] \mid[L]}{[R] \mid[Q]}\right] \quad\left[\mathcal{K}_{k}\right]=\left[\frac{\left[K_{k}\right]\left[\left[L_{k}\right]\right.}{\left[R_{k}\right] \mid\left[Q_{k}\right]}\right]
$$

Accordingly, the following equation must now be satisfied by $E_{k}$ :

$$
[K]\{U\}+[L]\{V\}=\sum_{k=1}^{N_{e}} E_{k}\left[K_{k}\right]\{U\}+\sum_{k=1}^{N_{e}} E_{k}\left[L_{k}\right]\{V\}=\{0\}
$$

It is assumed that the modulus is known (or a given relative value is imposed) over $N$ brick elements, with $N>0$. Thus, the $N_{e}-N$ modulus values that remain must satisfy: 


$$
\sum_{k=1}^{N_{e}-N} E_{k}\left\{\left[K_{k}\right]\{U\}+\left[L_{k}\right]\{V\}\right\}=-\sum_{l=1}^{N} \tilde{E}_{l}\left\{\left[K_{l}\right]\{U\}+\left[L_{l}\right]\{V\}\right\}
$$

$$
\Leftrightarrow[A]\{E\}=\{B\}
$$

where the $\tilde{E}_{k}$ are the values of the known or imposed moduli. Eventually, the size of matrix $[A]$ is $\left(3 N_{n}-N_{f}\right) \times\left(N_{e}-N\right)$. If $N=0,\{B\}$ is the null vector because the nonzero nodal force have been removed from the equations as they cannot be measured in practice. Therefore, one ends up with a singular system of equations. Consequently, at least one value of the moduli must be known or imposed $(N>0)$ for solving the equations and find a relevant solution. Nevertheless, in practice, increasing $N$ means decreasing the number of unknowns but keeping the same number of equations. Thus, the redundancy of the equations is increased and consequently, the filtering of noise will be improved. It will be shown further that robustness issues are crucial within the identification of modulus distribution. Therefore, it is recommended to use the largest possible value for $N$.

\subsubsection{Physical interpretation of the equations}

Eq. 2.10 can be interpreted quite simply in a one-dimensional (1D) solid discretized with spar elements as in Fig. 5.2. Spar elements have linear shape functions and each node has only one DoF: the translation in the direction parallel to the bar. This means that the only component of the strain tensor, denoted $\varepsilon$ here, and the only component of the stress tensor, denoted $\sigma$ here, are constant along each spar element.

The principle of Eq. 2.10 is to ensure that the equilibrium condition is satisfied, like in the so-called "equilibrium gap method" [Claire et al., 2004]. The equilibrium equations are particularly simple in 1D. The equilibrium equation means that the nodal force $F$ at node $x_{n}$ must be zero (Fig. 5.2). The nodal forces depend on the stress increment between the consecutive spar elements, which can be related to the increment of the displacement between two consecutive nodes. Therefore, the following equations can be developed:

$$
\begin{aligned}
F\left(x_{n}\right)=0 & \Rightarrow \frac{\sigma\left(X_{n+1}\right)-\sigma\left(X_{n}\right)}{X_{n+1}-X_{n}}=0 \\
& \Rightarrow \sigma\left(X_{n+1}\right)-\sigma\left(X_{n}\right)=0 \\
& \Rightarrow E\left(X_{n+1}\right) \varepsilon\left(X_{n+1}\right)-E\left(X_{n}\right) \varepsilon\left(X_{n}\right)=0 \\
& \Rightarrow E\left(X_{n+1}\right) \frac{u\left(x_{n+1}\right)-u\left(x_{n}\right)}{x_{n+1}-x_{n}}-E\left(X_{n}\right) \frac{u\left(x_{n}\right)-u\left(x_{n-1}\right)}{x_{n}-x_{n-1}}=0
\end{aligned}
$$

yielding:

$$
\left[u\left(x_{n+1}\right)-u\left(x_{n}\right)\right] E\left(X_{n+1}\right)-\left[u\left(x_{n}\right)-u\left(x_{n-1}\right)\right] E\left(X_{n}\right)=0
$$


The latter equations give a relationship between the modulus $E$ of two consecutive elements. It is the discretized form of the following differential equation:

$$
\frac{\partial}{\partial x}\left(E \frac{\partial u}{\partial x}\right)=E \frac{\partial^{2} u}{\partial x^{2}}+\frac{\partial E}{\partial x} \frac{\partial u}{\partial x}=0
$$

If Eq. 2.13 is developed across all the spar elements, one obtains a system of equations involving all the moduli of all the elements.

The derived system of equations is ill-conditioned because it is the discretized form of a differential equation involving a second-order differentiation of the displacement field (Eq. 2.14). Computing the second-order derivatives of noisy data yields poorly conditioned system of equations when it is solved numerically. Consequently, if a small error is added to the coefficients of the system, large errors result in the solution. Therefore, we expect this approach to be sensitive to noise.

Similar instability is also to be expected when solving Eq. 2.10 because it is the extrapolation of Eq. 2.13 into 3D. The difference is that, in Eq. 2.13, the equilibrium condition at each node yields three equations because the nodal force has three components: $F_{x}, F_{y}$ and $F_{z}$. In $3 \mathrm{D}$, each equation gives a relationship between the moduli of the eight finite elements surrounding each node. This results in a large system of equations that corresponds to the discretized form of three PDEs involving the first order derivatives of the displacement field. Regularizing approaches exist for improving the stability of the solution [Tikonov and Arsenin, 1977], but they are not investigated here because they require relevant a priori information which is not within the scope of this paper.

\subsection{General solution of the inverse problem}

The system of equations derived in Eq. 2.11 is an overdetermined system of equations. There are more equations than unknowns because the equilibrium condition at each node yields three equations. The resolution of this system of equations using weighted least squares was investigated in [Claire et al., 2004]. A resolution based on the virtual fields method is considred in this study.

\subsubsection{Modulus ratio identification}

For the sake of simplicity, let us consider the case where the investigated solid is composed of only two different materials. Thus, only two different values are possible for the modulus in each finite element. A value of one is prescribed for the finite elements which are known to be located in the first material, called the reference material. An unknown value, denoted $\check{E}$, is prescribed for the finite elements which are known to be located in the other material, called the unknown material. $\check{E}$ therefore represents the ratio between the modulus of both materials.

Accordingly, Eq. 2.11 can be modified to: 


$$
\check{E} \sum_{\text {unknown }}\left\{\left[K_{k}\right]\{U\}+\left[L_{k}\right]\{V\}\right\}=-\sum_{\text {reference }}\left\{\left[K_{k}\right]\{U\}+\left[L_{k}\right]\{V\}\right\}
$$

This overdetermined system of equations is solved by multiplying it with a suitable column vector, denoted $\left\{U^{*}\right\}$. This column vector features the nodal DoFs of a piecewise virtual displacement field [Toussaint et al., 2006]. Finally, the following solution is obtained:

$$
\check{E}=\frac{\left\{U^{*}\right\}^{t} \sum_{\text {unknown }}\left\{\left[K_{k}\right]\{U\}+\left[L_{k}\right]\{V\}\right\}}{\left\{U^{*}\right\}^{t} \sum_{\text {reference }}\left\{\left[K_{k}\right]\{U\}+\left[L_{k}\right]\{V\}\right\}}
$$

Algorithms have been developed in 2D for providing optimal virtual fields [Avril et al., 2004]. However, they require the inversion of large matrices which is computationally expensive when $3 \mathrm{D}$ data are processed. Therefore, an intuitive guess for vector $\left\{U^{*}\right\}$ is the simplest solution here. An example will be given in section 3 .

\subsubsection{Modulus distribution identification}

When the structure is composed of several different materials, or when the structure of the investigated solid is unknown, the system of equations in Eq. 2.11 must be solved directly without any simplifications, such as the one in Eq. 2.15.

In Eq. 2.11, the number of unknown modulus values is assumed as lower than the number of equations for ensuring uniqueness of the solution. This is the most likely situation as three independent equations are derived from the equilibrium written at each single node. This overdetermined system is solved by multiplying it with a suitable set of column vectors, featuring the nodal DoFs of piecewise virtual displacement fields [Toussaint et al., 2006]. A good choice from a numerical perspective [Avril and Pierron, 2007] is to use vectors that can be written in the form:

$$
\left\{U^{*}\right\}=[\alpha]\left\{\left[K_{k}\right]\{U\}+\left[L_{k}\right]\{V\}\right\}
$$

where $[\alpha]$ is a positive definite matrix and $k$ varies between 1 and $N_{e}-N$. The size of matrix $[\alpha]$ is $\left(3 N_{n}-N_{f}\right) \times\left(3 N_{n}-N_{f}\right)$.

Such a choice for the column vectors provides a system of equations that can be written:

$$
\left[[A]^{t}[\alpha][A]\right]\{E\}=[A]^{t}[\alpha]\{B\}
$$

where $[A]$ and $[B]$ have been defined in Eq. 2.11. $\left[[A]^{t}[\alpha]^{-1}[A]\right]$ is a large matrix that has the interesting property of being symmetric positive definite. As $[A]$ is also sparse if $[\alpha]$ is well chosen, iteratively solving Eq. 2.18 by the conjugate gradient method is now possible [Brodie, 1977]. This provides a significant computational acceleration, allowing, for example, one million modulus values to be calculated in less than 10 minutes. Therefore, the volume of interest can be refined in order to obtain an acceptable spatial resolution for the modulus variation across the volume of the investigated solid. 


\subsubsection{Derivation of matrix $[\alpha]$}

The solution of Eq. 2.18 can be interpreted as the weighted least-squares solution of equations 2.11, used for example in the "equilibrium gap method" [Claire et al., 2004]. It consists of minimizing the following cost function:

$$
\varphi(\{E\})=\frac{1}{2}\{[A]\{E\}-\{B\}\}^{t}[\alpha]\{[A]\{E\}-\{B\}\}
$$

where $[\alpha]$ is a matrix used to minimize the effect of noise in the data [Tarantola, 2005]. A larger weight is given to nodes and components where the confidence on the data is high and a lower weight where the confidence is small. The evaluation of the confidence level depends on the data themselves and it is different from one application to another. An example will be given in section 3 . Because $[\alpha]$ is usually a sparse matrix, the conjugate gradient method may be used to solve Eq. 2.18.

\subsection{Solution of the inverse problem for nearly incompressible materials}

Most human soft tissues are almost incompressible [Ophir et al., 2001]. Although our approach is applicable to all types of material, if the Poisson ratio is very close to 0.5 , some components of the Hooke matrix in Eq. 2.7 will tend towards infinity. Accordingly, the application of our approach to this example is somewhat more complex than the general case but it will be shown that this situation can still be handled.

For nearly incompressible materials, Poisson's ratio is close to 0.5. Accordingly, Eq. 2.7 can be modified into the following equation if the material is assumed incompressible linear elastic [Barbone and Gokhale, 2004]:

$$
\left\{\begin{array}{l}
\sigma_{x x} \\
\sigma_{y y} \\
\sigma_{z z} \\
\sigma_{x y} \\
\sigma_{x z} \\
\sigma_{y z}
\end{array}\right\}=\left\{\begin{array}{l}
p \\
p \\
p \\
0 \\
0 \\
0
\end{array}\right\}+2 G\left[\begin{array}{llllll}
1 & 0 & 0 & 0 & 0 & 0 \\
0 & 1 & 0 & 0 & 0 & 0 \\
0 & 0 & 1 & 0 & 0 & 0 \\
0 & 0 & 0 & 1 & 0 & 0 \\
0 & 0 & 0 & 0 & 1 & 0 \\
0 & 0 & 0 & 0 & 0 & 1
\end{array}\right]\left\{\begin{array}{l}
\varepsilon_{x x} \\
\varepsilon_{y y} \\
\varepsilon_{z z} \\
\varepsilon_{x y} \\
\varepsilon_{x z} \\
\varepsilon_{y z}
\end{array}\right\}
$$

where $p$ is the hydrostatic pressure variation and $G$ is the shear modulus.

In a solid, the hydrostatic pressure variation can be related to the relative volume variation $d V$ according to:

$$
p=\kappa \frac{d V}{V}
$$

where $\kappa$ is the bulk modulus, which is almost uniform in human tissues, close to the bulk modulus of water [Manduca et al., 2001].

The relative volume variation can be approximated to first order by the sum of the three diagonal components of the infinitesimal strain tensor: 


$$
\frac{d V}{V}=\operatorname{Tr}(\varepsilon)=\varepsilon_{x x}+\varepsilon_{y y}+\varepsilon_{z z}
$$

In nearly incompressible materials, $\operatorname{Tr}(\varepsilon)$ is negligible compared to any component $\varepsilon_{i j}$ of the strain tensor. Therefore, as both $\kappa$ and $\operatorname{Tr}(\varepsilon)$ have negligible variations across the volume of human tissues, the gradient of the hydrostatic pressure may be neglected. Such an assumption was actually proved to be too restrictive for nearly incompressible materials subjected to time-harmonic loadings at a frequency of $150 \mathrm{~Hz}$ [Park and Maniatty, 2006]. However, the effect of acceleration forces could account for the existence of non negligible gradients of the hydrostatic pressure in dynamics. Results shown further will prove that neglecting gradients of the hydrostatic pressure in quasi-static is relevant.

Assuming negligible gradients of the hydrostatic pressure, it can be shown that the hydrostatic pressure will not be involved in the equations. Indeed, let us consider a virtual displacement field, denoted $\boldsymbol{u}^{*}$, which is null over the whole boundary of the solid (like all the virtual displacement fields introduced previously). Integrating by parts the virtual work created by the hydrostatic pressure terms, one gets:

$$
\int_{\Omega}<\nabla \boldsymbol{u}^{*}>\left\{\begin{array}{l}
p \\
p \\
p \\
0 \\
0 \\
0
\end{array}\right\} \mathrm{d} V=-\int_{\Omega} \boldsymbol{u}^{*} \cdot \nabla p \mathrm{~d} V+p \int_{\partial \Omega} \boldsymbol{u}^{*} \mathrm{~d} S=0
$$

Therefore, $p$ is not involved in the virtual work for a virtual field that is null at the boundary. As this is the case for the virtual fields that yield Eq. 2.11 or Eq. 2.15, the hydrostatic pressure is not involved in Eq. 2.11 and Eq. 2.15. Thus, only the G-dependent part of the elementary stiffness matrix derived from Eq. 2.6 is needed to construct the global stiffness matrix, denoted $\left[\mathcal{K}^{\bullet}\right]$. The novel elementary stiffness matrix can be written:

$$
\left[\mathcal{K}_{k}^{\bullet}\right]=G_{k} \int_{e_{k}}[\nabla \Phi]\left[\nabla \Phi^{e_{k}}\right] \mathrm{d} V
$$

where $G_{k}$ is the shear modulus of the current finite element. Thus, the unknowns for the problem defined here will be the values of the shear modulus instead of the values of Young's modulus. For an incompressible material, the two are related through $E=3 G$. The vector of unknown shear moduli, denoted $\{G\}$, will satisfy similar equations to Eq. 2.11 and Eq. 2.15:

- for the modulus ratio identification:

$$
\check{G} \sum_{\text {unknown }}\left\{\left[K_{k}^{\bullet}\right]\{U\}+\left[L_{k}^{\bullet}\right]\{V\}\right\}=-\sum_{\text {reference }}\left\{\left[K_{k}^{\bullet}\right]\{U\}+\left[L_{k}^{\bullet}\right]\{V\}\right\}
$$


- for the shear modulus distribution:

$$
\sum_{k=1}^{N_{e}-n} G_{k}\left\{\left[K_{k}^{\bullet}\right]\{U\}+\left[L_{k}^{\bullet}\right]\{V\}\right\}=-\sum_{l=1}^{n} \tilde{G}_{l}\left\{\left[K_{l}^{\bullet}\right]\{U\}+\left[L_{l}^{\bullet}\right]\{V\}\right\}
$$

The only differences compared with Eq. 2.11 and Eq. 2.15 come from the different definition of stiffness matrices $\left(\left[\mathcal{K}_{k}^{\bullet}\right]\right.$ from Eq. 2.24 rather than $\left[\mathcal{K}_{k}\right]$ from Eq. 2.6).

\section{Application to experimental data}

\subsection{Experimental techniques and specimen}

Our approach was applied to the experimental data presented by [Steele et al., 2000]. The experiments were performed on a phantom containing a inclusion four times stiffer than the background. The specimen has a $80 \times 64 \times 154 \mathrm{~mm}^{3}$ parallelepipedic shape and the inclusion is a $25 \mathrm{~mm}$ diameter sphere (Figure 5.3). Semicosil 921 silicone gel (Wacker Silicones Corporation, Adrian, MI) was used to construct the phantom qualitatively simulating the mechanical properties of soft human tissues. It was checked using standard uniaxial tension [Steele et al., 2000] that this material has a linear elastic isotropic behaviour and is nearly incompressible.

The phantom was placed between two acrylic plates in a pneumatically driven device. A vertical deformation of approximately $6 \mathrm{~mm}$, or about $7.5 \%$ average strain, was applied. A stimulated echo MRI sequence using displacement encoding gradient pulses was employed to measure the phantom's internal static displacements [Chenevert et al., 1998]. The purpose of this paper is not to present this technique which was detailed in [Steele et al., 2000]. Let us just give the following basics about MRI.

MRI is an imaging modality which is primarily used to construct pictures of the Nuclear Magnetic Resonance (NMR) signal from the hydrogen atoms in an object. NMR is a fundamental property of nature possessed by protons, which is induced by pulses of magnetic field applied to the object. Varying gradients of magnetic fields, each voxel of the object can be encoded with different frequencies and phase angles. Therefore, the spectrum of the NMR signal collected by a receptor (coil) is affected by the applied gradients. A simple Fourier transform is capable of positioning the signal in the right voxel. However, the Fourier transform of a real number is a complex number. The magnitude of this complex number (magnitude of the MR signal) usually provides nice maps of the object topography [MacRobbie et al., 2003]. The phase angle of this complex number can be used as well to measure motions like blood flows [MacRobbie et al., 2003] or displacement fields [Steele et al., 2000]. It requires particular pulses of the magnetic fields, like the ones used in the sequence presented in [Steele et al., 2000].

Using this sequence, $256 \times 256 \times 64$ matrices of data were obtained, covering a $110 \times 80 \times 75 \mathrm{~mm}^{3}$ field of view. Only a $50 \times 50 \times 42 \mathrm{~mm}^{3}$ field of view, covered by a grid of $116 \times 142 \times 37$ voxels, was kept around 
the inclusion because the data outside this volume were missing or were too noisy. The resulting phase maps in this $50 \times 50 \times 42 \mathrm{~mm}^{3}$ region of interest were unwrapped using a temporal unwrapping approach explained by Huntley and Saldner [Huntley and Saldner, 1993]. Once unwrapped, the phase maps were multiplied by the respective sensitivity factors in order to get the displacement maps (Figure 5.4). Using high pass filters, the uncertainty of the displacement fields induced by the measurement noise was assessed. It is about $0.03 \mathrm{~mm}$.

A $9 \times 9 \times 1$ median filter kernel (only averaging within the slices) was applied to the unwrapped phase maps for reducing the noise level and eliminating inconsistent values (outliers). The uncertainty of displacements after filtering is about $0.004 \mathrm{~mm}$.

\subsection{Constitutive equations for the tested material}

The $50 \times 50 \times 42 \mathrm{~mm}^{3}$ volume of interest was meshed using $53 \times 66 \times 36$ brick-shaped finite elements for the identification. The DoFs at the nodes are identified by least-squares regression. These DoFs are used to derive the strain values at the centre of each of the $53 \times 66 \times 36$ brick-shaped finite elements by using a centred finite difference scheme. One obtains a $3 \mathrm{D}$ array of $53 \times 66 \times 36$ mapping a volume of $50 \times 50 \times 42 \mathrm{~mm}^{3}$. Therefore, the "strain gauge volume" is $0.94 \times 0.76 \times 1.2 \mathrm{~mm}^{3}$. However, this is without taking account of the $9 \times 9 \times 1$ median filter which was applied onto the initial $116 \times 142 \times 37$ array of displacement data. Taking account of it, the gauge volume is actually: $4.2 \times 3.4 \times 1.2 \mathrm{~mm}^{3}$. Within each of these volumes, strains are measured with an uncertainty of about 0.004 . This uncertainty has been deduced from the uncertainty of displacements given in the previous section.

The obtained $\varepsilon_{x x}$ and $\varepsilon_{y y}$ fields, as well as the sum of the three diagonal components of the strain tensor: $\varepsilon_{x x}+\varepsilon_{y y}+\varepsilon_{z z}=\operatorname{Tr}(\varepsilon)$ have been plotted across a given cross section at $x=0$ in Figure 5.5. The material investigated in this study is almost incompressible. Indeed, for the example addressed here, it can be checked that $\operatorname{Tr}(\varepsilon)$ is negligible compared to $\varepsilon_{x x}$ or $\varepsilon_{y y}$. Only noise is remaining in the field $\operatorname{Tr}(\boldsymbol{\varepsilon})$, which has the same range order as the strain uncertainty, estimated at about 0.004 . Due to this property, it can be assumed that the investigated material is incompressible. Thus, its Poisson's ratio is close to 0.5 and the modified approach described in Section 2.3 can be applied.

\subsection{Identification of the stiffness ratio between both media}

The $50 \times 50 \times 42 \mathrm{~mm}^{3}$ volume of interest was meshed using $53 \times 66 \times 36$ brick-shaped finite elements for the identification. The DoFs at the nodes are identified by least-squares regression.

The elements located inside the inclusion have been detected using the magnitude of the same MR signal as the one used for measuring the displacement fields. The magnitude of an MR image depends primarily on $T_{1}, T_{2}$, and proton density, and is generally not related to the mechanical properties of the 
imaged object [MacRobbie et al., 2003]. However, in the magnitude images (Figure 5.6) the inclusion is clearly seen due to the inherent $T_{1}$ and $T_{2}$ contrast of the stimulated echo sequence.

A Matlab function has been developed for estimating an ellipsoidal contour round a 3D region of distinctly higher or lower signal strength (magnitude of the MR signal in this case) than that in the background region of interest. This contour is characterized by six geometric parameters $\left(x_{0}, y_{0}\right.$ and $z_{0}$ for the center, $r_{x}, r_{y}$ and $r_{z}$ for the size) defining the ellipsoid as those pixels for which:

$$
\frac{\left(x-x_{0}\right)^{2}}{r_{x}^{2}}+\frac{\left(y-y_{0}\right)^{2}}{r_{y}^{2}}+\frac{\left(z-z_{0}\right)^{2}}{r_{z}^{2}} \leq 1
$$

An ellipsoidal description of the inclusion is used instead of a spherical one because the voxels do not have a cubic shape (the $50 \times 50 \times 42 \mathrm{~mm}^{3}$ field of view is covered by a grid of $116 \times 142 \times 37$ voxels). By applying this Matlab function to the magnitude field, all the finite elements which have their centroid located inside the inclusion have been detected. This information is used to complete Eq. 2.16 where the "reference" becomes the background material and the "unknown" becomes the inclusion. Accordingly, the ratio can be written:

$$
\check{G}=\frac{\left\{U^{*}\right\}^{t} \sum_{\text {inclusion }}\left\{\left[K_{k}^{\bullet}\right]\{U\}+\left[L_{k}^{\bullet}\right]\{V\}\right\}}{\left\{U^{*}\right\}^{t} \sum_{\text {outside }}\left\{\left[K_{k}^{\bullet}\right]\{U\}+\left[L_{k}^{\bullet}\right]\{V\}\right\}}
$$

The components of vector $\left\{U^{*}\right\}$ are defined across the whole measurement volume. Accordingly, they are defined as a polynomial function. They have been chosen as:

$$
\boldsymbol{u}^{*}=\left\{\begin{array}{l}
0 \\
(x-L / 2)(x+L / 2)(y-l / 2)(y+l / 2)(z-h / 2)(z+h+2) \\
0
\end{array}\right\}
$$

This choice has been made for the following reasons:

- the virtual field is zero across the whole contour of the volume of interest, which makes the identification insensitive to the unknown distribution of tractions at the boundary because all the nonzero components of vector $\{b\}$ in Eq. 2.9 are multiplied by zero with such a choice of $\left\{U^{*}\right\}$.

- only $u_{y}^{*}$ is nonzero $\left(u_{x}^{*}=u_{z}^{*}=0\right)$. It means that the $x$ and $z$ components of nodal forces are multiplied by zero in the equation of the principle of virtual work. This is equivalent to saying that the $x$ and $z$ components of nodal forces are not considered. Consequently, only the $y$ component of nodal forces is considered in the equations. If one refers to the equilibrium equations, the $y$ component of nodal forces is the only of the three classical equilibrium equations to involve $\sigma_{y y}$. As $y$ is the direction of loading, $\sigma_{y y}$ is the largest stress component. Therefore, it is the one that provides the highest sensitivity to the modulus variations. The two other equilibrium equations involve marginal stress components in this test and this is the reason why they are not considered. 
- Among all the virtual fields that verify the two previous conditions, the one which has the lowest gradients is sought, according to the study of noise sensitivity presented in [Avril et al., 2004]. The polynomial function which satisfies the two previous conditions and which has the lowest gradients may be written as in Eq. 3.3 (polynomial with the lowest possible order).

When applied to the experimental results with $53 \times 66 \times 36$ brick elements and the previous virtual field (Eq. 3.3), Eq. 3.2 gives: $\check{G}=4.2$. This is in agreement with the reference value of 4 (Tab. 5.2) and therefore appears very promising for further applications of our approach.

The remaining difference may be due to measurement errors and the assumptions made here (the main ones being: neglecting the gradient of the hydrostatic pressure and neglecting geometric nonlinearities induced by the large deformation [Skovoroda et al., 1999]). Moreover, the accuracy of the detection of the boundary separating the two materials also affects the results. An error of $1 \%$ in the diameter of the inclusion results in an error of $20 \%$ in the modulus ratio. Therefore, even if the possibility to characterize this ratio is a real asset, it can only be used to complete the information provided by the modulus distribution, as shown in the following section.

\subsection{Identification of the modulus distribution}

The $50 \times 50 \times 42 \mathrm{~mm}^{3}$ volume of interest was again meshed using $53 \times 66 \times 36$ brick-shaped finite elements. Here, the modulus was assumed to be known only on the boundary of the volume of interest. The same assumption was used by [Steele et al., 2000]. Thus, $51 \times 64 \times 34=110976$ values are unknown inside the volume of interest. The modulus of the boundary elements was set to unity, i.e. $\left\{\tilde{G}_{l}\right\}$, to get a relative value of the moduli inside the specimen.

500 iterations of the conjugate gradient method were used to solve the system of equations ( 2 minutes using a Pentium M processor, $1400 \mathrm{MHz}$ ).

The $[\alpha]$ matrix was chosen to give zero weight to the nodal forces in the $x$ and $z$ directions and uniform weight to the nodal forces in the $y$ direction. We used a zero weight in the $x$ and $z$ directions because we wanted to involve only $\sigma_{y y}$ in the equations. As $y$ is the direction of loading, $\sigma_{y y}$ is the component of stresses for which the noise affects the least the equations. The uniform weight in the $y$ direction has been chosen because the equilibrium equation that involves the $\sigma_{y y}$ component is assumed to have the same reliability everywhere across the field.

Reconstruction results are presented in Figure 5.7a. As there are 36 finite elements along the $z$ direction, each plane passing through one of the centroids of these finite elements represents a cross section where a $53 \times 66$ matrix of results is available. All of the $53 \times 66$ result matrices across all 36 cross-sectional planes are plotted in the same picture, starting from the $x=-20.4 \mathrm{~mm}$ plane on the top left to the $x=20.4 \mathrm{~mm}$ plane on the bottom right in Fig. 5.7. This plotting scheme allows one to visualize the three-dimensional results. 
It can be noticed in the results that the highest modulus values delimit a volume (Figure 5.7a) that is similar to the actual volume of the inclusion (Figure 5.7b). The reference region occupied by the inclusion in Figure 5.7b has been determined from the magnitude of the MR signal. Tab. 5.1 presents the reference ellipsoid boundaries identified from the signal magnitude and the reconstructed modulus distribution. These ellipsoids are similar within a voxel. This proves that the identified modulus distribution is in close agreement with the actual one from a qualitative point of view.

Concerning the quantitative results, the average value of the modulus identified in the surrounding material is 0.69 and the average value of the modulus identified in the inclusion is 2.98 . Thus the ratio between both modulus values is 4.3. This is in agreement with the ratio of 4.2 identified in Section 3.3. This is also in agreement with the reference value of 4 (Tab. 5.2)

The main source of errors in Fig. 5.7a seems to be the noise in the experimental data. The standard deviation of the results is 1 in the region of the inclusion and 0.4 in the surrounding material. This means that the coefficient of variation (ratio between the standard deviation and the average) is $33 \%$ in the region of the inclusion and $57 \%$ in the surrounding material. It is worth recalling that the results shown in Figure 5.7a were not obtained from raw displacement data, but from data filtered using a median filter with a kernel size of $9 \times 9 \times 1$. The effect of filtering on the reconstruction has been investigated and results are reported in Tab 5.3. Different kernel sizes were tried out, ranging from $1 \times 1 \times 1$ (no filtering) up to $10 \times 10 \times 1$. For each set of filtered data, the same identification algorithm based on the virtual fields method was applied for reconstructing the modulus distribution. From the reconstructed modulus distribution, for all the different tested filtering kernel sizes, the boundary of the inclusion was traced and the average of the modulus both in the inclusion and around was derived. Then the ratio of both averages was computed. It was shown that the identified ratio varies. It first increases and then stabilizes for kernel size larger than $4 \times 4 \times 1$. It means the median filtering with a kernel size $9 \times 9 \times 1$ (the one used to provide the distribution of Figure 5.7 a) does not disturb the accurate detection of the edges of the inclusion. But it provides a less scattered distribution than kernel size $4 \times 4 \times 1$ (see the standard deviations reported in Tab 5.3), justifying its use in practice.

Although a value of 1 has been imposed to the boundary, only an average value of 0.69 is found in the surrounding material. Actually, in Fig. 5.7a, it can be noticed that the modulus distribution in the background is not homogeneous. Low values of the modulus (below the average value of 0.69) alternate with higher values of the modulus (above the average value of 0.69). These artifacts, which look like "waves" enveloping the inclusion in Fig. 5.7(a) are likely to be numerical. Indeed, the system of equations 2.18 is only solved approximately here using the conjugate gradient method. The conjugate gradient method is interrupted after only 500 iterations so as to reduce noise in the solution, because the fewer the number of iterations, the smoother the solution. Accordingly, 500 iterations was found as a good compromise between this smoothing effect and the convergence of the algorithm. For a number of iterations ranging from 100 to 1000, the modulus distribution was reconstructed and the modulus 
averages and standard deviations were derived both in the inclusion and in the surrounding material (Tab 5.4). It was shown that the identified averages vary, and so do the standard deviations. The ratio of both averages first increases and then stabilizes for a number of iterations larger than 500. But increasing the number of iterations beyond 500 still increases the standard deviation. Therefore, 500 can be considered here as the optimal number of iterations.

\section{Discussion}

\subsection{Analysis of the sensitivity to noise}

The scatter in the reconstruction results is likely induced by the noise amplification of the ill-conditioned equations 2.26. As explained in Section 2.1.5, a small error in the coefficients of the matrix can induce large errors in the results [Tarantola, 2005].

To evaluate the noise amplification a model of a homogeneous solid having the same size as the one investigated here was subjected to a uniaxial tensile test. Thus, $\varepsilon_{x x}, \varepsilon_{y y}$ and $\varepsilon_{z z}$ are constant, with $\varepsilon_{y y}=\varepsilon, \varepsilon_{x x}=\varepsilon_{z z}=-0.5 \varepsilon$ and $\varepsilon_{x y}=\varepsilon_{x z}=\varepsilon_{y z}=0$.

Those values were directly prescribed at the centroid of each finite element in order to build up the system of equations 2.26. Then, a Gaussian white noise (Standard Normal distribution), multiplied by a scaling factor of $\eta=\varepsilon / 100$, was added to all the components of the strain tensor. The system of equations of Eq. 2.26 was then solved with the noisy values of the strain tensor.

The resulting modulus variation identified using this system of equations is similar to white noise (Figure 5.8), with a standard deviation of 0.22 . This means that the amplification factor is about 20 between the coefficient of variation of noise in the strain fields and the coefficient of variation of the relative modulus error.

The average strain in the direction of loading is $7.5 \%$, which means that the solid could not be more deformed within its elastic range. Therefore, it is not possible to decrease the coefficient of variation of noise in the strain fields. One has to focus on the amplification factor to decrease the scattering of the results.

Smoothing the results appeared as the most efficient way of removing the noise. A $5 \times 5 \times 1$ median filter kernel (only averaging within the slices) was applied to obtain the results shown in Figure 5.9. The amplification factor was divided by 5 , which looks evident in the figure. After smoothing, the ratio between the average value of the modulus identified in the surrounding material and the average value of the modulus identified in the inclusion is still 4.3, which means that the median filter does not bias the results. Heterogeneities can still be detected within the inclusion. They may actually exist in the real material, or just be artifacts caused by some assumptions made in this study. It is yet curious that similar heterogeneities have already been recovered for a similar phantom using a different approach of reconstruction [Park and Maniatty, 2006]. 


\subsection{Comparison with the strain imaging approach}

The distribution of the strain component in the loading direction $\left(\varepsilon_{y y}\right)$ can be used as an indicator for the modulus distribution when the stress distribution is almost homogeneous [Doyley et al., 2005]. According to [Ophir et al., 2001], for low modulus contrast and simplified geometrical boundary conditions, the strain distribution may be a relatively good representation of the underlying modulus distribution. This approach for determining the modulus distribution is called the "strain imaging" approach. Let us show that it is inappropriate here.

According to the "strain imaging" approach [Ophir et al., 2001], the ratio $\sigma / \varepsilon_{y y}$ should represent

the distribution of the Young modulus across the volume. As $\sigma$ is unknown here, the ratio $\varepsilon_{y y}^{\text {average }} / \varepsilon_{y y}$ has been computed across the whole volume and plotted for all the cross sections along the $z$ direction (similarly as in Figure 5.7). The results are shown in Figure 5.10. They are less noisy than the ones of our approach because they are obtained by a mere first order differentiation. However, errors occur, that prevent the strain imaging approach from being useful either in a quantitative way or in a qualitative way for the example addressed here. Quantitatively, the modulus of the inclusion is significantly underestimated (average of 2.5 instead of 4). Qualitatively, the location of the inclusion is well found but its boundary is blurred in the plane $z=0$. Moreover, other heterogeneities are erroneously detected at the top of the volume of interest. These result from the existence of friction forces at these locations.

Due to this double failure, it can be concluded that the example processed here can only be addressed satisfactorily using an inverse method like our approach or like the one developed by Skovodora et al. [Skovoroda et al., 1995].

\subsection{Comparison with Skovodora's approach [Skovoroda et al., 1995]}

Steele et al. [Steele et al., 2000] have processed the same experimental data using another direct approach to determine the distribution of the shear modulus. Their approach is similar to ours in principle, but instead of the nodal forces, the curl of the nodal forces is cancelled in their approach. To remove the hydrostatic pressure from the equilibrium equations, it was assumed in Section 3.2 that its gradient is negligible and that using particular virtual fields which are null on the boundary cancelled its contribution (see Eq. 2.23). Computing the curl of the nodal forces is another method for ridding the hydrostatic pressure from the equilibrium equations. Eventually, one obtains a hyperbolic PDE with the shear modulus values as unknowns [Barbone and Gokhale, 2004, Skovoroda et al., 1995, Skovoroda et al., 1998, Skovoroda et al., 1999]. But the derivation of these differential equations is based on a thirdorder differentiation of the data instead of the second-order one in our case (Eq. 2.14). According to the discussion in Section 2.1.5, this third-order differentiation brings even more instability. The instability is tackled by the authors via numerical integration of the equations [Skovoroda et al., 1995]. This yields 
a system of equations similar to the one of Eq. 2.26, but the coefficients of their matrix involve the second order derivatives of the data whereas ours involve only the first order derivatives.

The poor stability of the equations solved by Steele et al. may explain the artifacts encountered in their results [Steele et al., 2000]. Our results show that even when there is one order less in the differentiation, results are still very noisy (Figure 5.7). Then, only filtering (see Figure 5.9) or regularization approaches based on relevant a priori information [Tikonov and Arsenin, 1977] can improve the stability.

The stability which is gained in our approach compared to Skovodora's one [Skovoroda et al., 1995] relies on the assumption that the gradient of the hydrostatic pressure can be neglected (Eq. 2.23). This assumption has already been used for time harmonic excitation at $150 \mathrm{~Hz}$ by [Park and Maniatty, 2006]. They actually showed that it tends to provide underestimated values. However, our results, which were obtained by neglecting the gradients of the hydrostatic pressure, are in agreement with the expected values. The gradients of the hydrostatic pressure are actually less critical in quasi-static studies than in dynamic studies. Accordingly, due to the relative uniformity of the bulk modulus in human tissues [Manduca et al., 2001] and due to their incompressibility, it may be justified to consider that the hydrostatic pressure is constant across quasi-statically deformed human tissues.

\section{Conclusion}

In this paper, the first extension of the virtual fields method to 3D bulk full-field measurements has been presented. It has been tested on displacement fields measured by stimulated echo MRI on a silicone gel phantom containing a stiff spherical inclusion. The identified ratio between the modulus of the inclusion and the modulus of the surrounding material is in close agreement with the reference value. Furthermore, the inclusion location is correctly determined. The range of modulus values is very scattered in the raw results, but smooth modulus distributions are easily recovered by filtering the noise.

The main limitations of the approach presented in this paper are the assumptions required for its application: linear elastic and isotropic behaviour, constant Poisson's ratio, quasi-static loading. The objective is now to overcome those limitations. Regarding the quasi-static requirement, it could be overcome by introducing in the equations both the hydrostatic pressure [Park and Maniatty, 2006] and the viscosity [Giraudeau and Pierron, 2005]. Nonetheless, this would permit faster loading-unloading cycles as the quasi-static requirement would no longer be needed. A drastic reduction of the data acquisition time (which was $6 \mathrm{~h}$ for the data processed here [Steele et al., 2000]) could then be envisaged. 


\section{Acknowledgements}

We are grateful to René Doucet, Head of ENSAM (Châlons en Champagne, France) who bestowed a leave upon Stéphane Avril for the achievement of this research project, and to EPSRC for partial support. J.M. Huntley is also grateful to the Royal Society and Wolfson Foundation for a Royal Society - Wolfson Research Merit Award. 


\section{References}

[Avril et al., 2004] Avril, S., Grédiac, M., and Pierron, F. (2004). Sensitivity of the virtual fields method to noisy data. Computational Mechanics, 34(6):439-452. Springer.

[Avril and Pierron, 2007] Avril, S. and Pierron, F. (2007). General framework for the identification of constitutive parameters from full-field measurements in linear elasticity. International Journal of Solids and Structures, 44:49785002. Springer.

[Barbone and Gokhale, 2004] Barbone, P. and Gokhale, N. (2004). Elastic modulus imaging: on the uniqueness and nonuniqueness of the elastography inverse problem in two dimensions. Inverse Problems, 20:283-296. Institute of Physics Publishing.

[Barbone and Oberai, 2007] Barbone, P. and Oberai, A. (2007). Elastic modulus imaging: some exact solutions of the compressible elastography inverse problem. Physics in Medicine and Biology, 52:1577-1593. Institute of Physics Publishing.

[Bonnet and Constantinescu, 2005] Bonnet, M. and Constantinescu, A. (2005). Inverse problems in elasticity. Inverse problems, 21:R1-R50. Institute of Physics Publishing.

[Brodie, 1977] Brodie, K. (1977). The State of the Art in Numerical Analysis. Ed. D. A. E. Jacobs, London: Academic Press.

[Bui, 1994] Bui, H. (1994). Inverse Problems in the Mechanics of Materials: An Introduction. CRC Press, Boca Raton, FL.

[Chenevert et al., 1998] Chenevert, T., Skovoroda, A., O’Donnell, M., and Emelianov, S. (1998). Elasticity reconstructive imaging by means of stimulated echo MRI. Magnetic Resonance in Medicine, 39:482-490. John Wiley and Sons, Ltd.

[Claire et al., 2004] Claire, D., Hild, F., and Roux, S. (2004). A finite element formulation to identify damage fields. Int. J. Numer. Methods Eng., 61:189-208. John Wiley and Sons, Ltd.

[Doyley et al., 2005] Doyley, M., Srinivasan, S., Pendergrass, S., Wu, Z., and Ophir, J. (2005). Comparative evaluation of strain-based and model-based modulus elastography. Ultrasound in Med. and Biol., 31(6):787-802. Elsevier.

[Fehrenbach et al., 2006] Fehrenbach, J., Masmoudi, M., Souchon, R., and Trompette, P. (2006). Detection of small inclusions by elastography. Inverse problems, 22:1055-1069. Institute of Physics Publishing.

[Giraudeau and Pierron, 2005] Giraudeau, A. and Pierron, F. (2005). Identification of stiffness and dampingproperties of thin isotropic vibratingplates usingthe virtual fields method: theory and simulations. Journal of Sound and Vibration, 284:757-781. Elsevier.

[Grédiac, 1989] Grédiac, M. (1989). Principe des travaux virtuels et identification. Comptes Rendus de l'Académie des Sciences, 309:1-5. Elsevier, in French with abridged English Version.

[Grédiac et al., 2006] Grédiac, M., Pierron, F., Avril, S., and Toussaint, E. (2006). The virtual fields method for extractiong constitutive parameters from full-field measurements: a review. Strain, 42:233-253. Blackwell.

[Huntley and Saldner, 1993] Huntley, J. and Saldner, H. (1993). Temporal phase-unwrapping algorithm for automated interferogram analysis. Applied Optics, 32:3047-3052. Optical Society of America.

[Liew and Pinsky, 2005] Liew, H. and Pinsky, P. (2005). Recovery of shear modulus in elastography using an adjoint method with b-spline representation. Finite Elements in Analysis and Design, 41:778-799. Elsevier.

[MacRobbie et al., 2003] MacRobbie, D., Moore, E., Graves, M., and Prince, M. (2003). MRI: From Picture to Proton. Cambridge University Press.

[Manduca et al., 2001] Manduca, A., Oliphant, T., Dresner, M., Mahowald, J., Kruse, S., Amromin, E., Felmlee, J., Greenleaf, J., and Ehman, R. (2001). Magnetic resonance elastography: Non-invasive mapping of tissue elasticity. Medical Image Analysis, 5:237-254. Elsevier.

[McLaughlin and Yoon, 2004] McLaughlin, J. and Yoon, J. (2004). Unique identifiability of elastic parameters from time-dependent interior displacement measurement. Inverse Problems, 20:25-45. Institute of Physics Publishing.

[Ophir et al., 2001] Ophir, J., Kallel, F., Varghese, T., Konofagou, E., Alama, S., Krouskop, T., Garra, B., and Righetti, R. (2001). Optical and acoustical imaging of biological media: Elastography. C. R. Acad. Sci. Paris, 2, Srie IV:11931212.

[Park and Maniatty, 2006] Park, E. and Maniatty, A. (2006). Shear modulus reconstruction in dynamic elastography: time harmonic case. Phys. Med. Biol., 51:3697-3721.

[Romano et al., 2001] Romano, A., Shirron, J., and Bucaro, J. (2001). On the noninvasive determination of material parameters from a knowledge of elastic displacements: Theory and numerical simulation. IEEE Transactions on Ultrasonics, Ferroelectrics, and Frequency Control, 45(3):751-759. IEEE Transactions, Journals, and Letters.

[Skovoroda et al., 1995] Skovoroda, A., Emelianov, S., and O'Donnell, M. (1995). Tissue elasticity reconstruction based on ultrasonic displacement and strain images. IEEE Transactions on Ultrasonics, Ferroelectrics, and Frequency Control, 42(4):747-765. IEEE Transactions, Journals, and Letters.

[Skovoroda et al., 1998] Skovoroda, A., Lubinski, M., Emelianov, S., and O'Donnell, M. (1998). Non linear estimation of the lateral displacement using tissue incompressibility. IEEE Transactions on Ultrasonics, Ferroelectrics, and Frequency Control, 45(3):491-503. IEEE Transactions, Journals, and Letters.

[Skovoroda et al., 1999] Skovoroda, A., Lubinski, M., Emelianov, S., and O'Donnell, M. (1999). Reconstructive elasticity imaging for large deformation. IEEE Transactions on Ultrasonics, Ferroelectrics, and Frequency Control, 46(3):523535. IEEE Transactions, Journals, and Letters.

[Steele et al., 2000] Steele, D., Chenevert, T., Skovoroda, A., and Emelianov, S. (2000). Three-dimensional static displacement, stimulated echo NMR elasticity imaging. Phys. Med. Biol., 45:1633-1648. Institute of Physics Publishing. 
[Tarantola, 2005] Tarantola, A. (2005). Inverse Problem Theory. SIAM.

[Tikonov and Arsenin, 1977] Tikonov, A. and Arsenin, V. (1977). Solutions of Ill-Posed Problems. Wiley, New York. [Toussaint et al., 2006] Toussaint, E., Grédiac, M., and Pierron, F. (2006). The virtual fields method with piecewise virtual fields. International Journal for Mechanical Sciences, 48(3):256-264. Elsevier.

[Weaver et al., 2005] Weaver, J., Doyley, M., Cheung, Y., Kennedy, F., Madsen, E., VanHouten, E., and Paulsen, K. (2005). Imaging the shear modulus of the heel fat pads. Clinical Biomechanics, 20:312-319. Elsevier.

[Zienkiewicz, 1977] Zienkiewicz, O. (1977). The finite element method. Mc Graw-Hill. 


\section{List of Tables}

5.1 Comparison of the inclusion contour (defined in Eq. 3.1) identified from the magnitude of the MR signal and from the identified modulus distribution . . . . . . . . . . . 24

5.2 Comparison of the identified ratios with the reference $\ldots \ldots \ldots \ldots$

5.3 Comparison of the results obtained with different sizes of the median filter kernel. . . 26

5.4 Comparison of the results obtained with different numbers of iterations in the conjugate gradient method. . . . . . . . . . . . . . . . . . . . 27 


\begin{tabular}{|c|c|c|c|c|c|c|}
\hline & $x_{0}$ (voxels) & $y_{0}$ (voxels) & $z_{0}$ (voxels) & $r_{x}$ (voxels) & $r_{y}$ (voxels) & $r_{z}$ (voxels) \\
\hline using the magnitude of the MR signal & 31.00 & 27.10 & 19.61 & 21.27 & 13.80 & 13.40 \\
\hline using the identified modulus distribution & 31.46 & 27.03 & 19.58 & 20.96 & 12.76 & 12.85 \\
\hline
\end{tabular}

Table 5.1. Comparison of the inclusion contour (defined in Eq. 3.1) identified from the magnitude of the MR signal and from the identified modulus distribution 


\begin{tabular}{|l|c|}
\hline ratio identified with a unique virtual field & 4.2 \\
\hline ratio identified from the fully reconstructed modulus distribution & 4.3 \\
\hline reference ratio from [Steele et al., 2000] & 4 \\
\hline
\end{tabular}

Table 5.2. Comparison of the identified ratios with the reference 


\begin{tabular}{|c|c|c|c|c|c|c|c|c|c|c|}
\hline $\begin{array}{l}\text { size of the median } \\
\text { filter kernel }\end{array}$ & $\begin{array}{c}1 \times 1 \times 1 \\
\text { (no filter) }\end{array}$ & $2 \times 2 \times 1$ & $3 \times 3 \times 1$ & $4 \times 4 \times 1$ & $5 \times 5 \times 1$ & $6 \times 6 \times 1$ & $7 \times 7 \times 1$ & $8 \times 8 \times 1$ & $9 \times 9 \times 1$ & $10 \times 10 \times 1$ \\
\hline $\begin{array}{l}\text { average modulus } \\
\text { identified in the inclusion }\end{array}$ & 2 & 2.3 & 2.5 & 2.8 & 2.8 & 2.9 & 3 & 3 & 3 & 3 \\
\hline $\begin{array}{l}\text { average modulus } \\
\text { identified in } \\
\text { the background }\end{array}$ & 0.64 & 0.61 & 0.62 & 0.65 & 0.67 & 0.68 & 0.69 & 0.7 & 0.7 & 0.7 \\
\hline $\begin{array}{l}\text { ratio between } \\
\text { both averages }\end{array}$ & 3.1 & 3.7 & 4.0 & 4.3 & 4.4 & 4.3 & 4.4 & 4.4 & 4.3 & 4.3 \\
\hline $\begin{array}{l}\text { standard deviation of } \\
\text { modulus values identified } \\
\text { in the inclusion }\end{array}$ & 2.8 & 2.5 & 2.2 & 1.9 & 1.8 & 1.6 & 1.5 & 1.3 & 1.3 & 1.2 \\
\hline $\begin{array}{l}\text { standard deviation of } \\
\text { modulus values identified } \\
\text { in the background }\end{array}$ & 1.2 & 0.6 & 0.6 & 0.5 & 0.5 & 0.4 & 0.4 & 0.4 & 0.4 & 0.4 \\
\hline
\end{tabular}

Table 5.3. Comparison of the results obtained with different sizes of the median filter kernel. 


\begin{tabular}{|l|c|c|c|c|c|}
\hline $\begin{array}{l}\text { number of iterations } \\
\text { in the conjugate gradient method }\end{array}$ & 100 & 250 & 500 & 750 & 1000 \\
\hline $\begin{array}{l}\text { average modulus } \\
\text { identified in the inclusion }\end{array}$ & 1.3 & 2.9 & 3.0 & 3.0 & 3.0 \\
\hline $\begin{array}{l}\text { average modulus } \\
\text { identified in the background }\end{array}$ & 0.7 & 0.7 & 0.7 & 0.7 & 0.7 \\
\hline $\begin{array}{l}\text { ratio between } \\
\text { both averages }\end{array}$ & 1.9 & 4.2 & 4.3 & 4.3 & 4.3 \\
\hline $\begin{array}{l}\text { standard deviation of modulus values } \\
\text { identified in the inclusion }\end{array}$ & 0.3 & 0.8 & 1.3 & 1.6 & 1.8 \\
\hline $\begin{array}{l}\text { standard deviation of modulus values } \\
\text { identified in the background }\end{array}$ & 0.3 & 0.3 & 0.4 & 0.4 & 0.45 \\
\hline
\end{tabular}

Table 5.4. Comparison of the results obtained with different numbers of iterations in the conjugate gradient method. 


\section{List of Figures}

5.1 Solid of any shape loaded on its boundary. . . . . . . . . . . . . . . . . . . . . . . . . . 29

$5.21 \mathrm{D}$ solid bar divided in spar finite elements (for the physical interpretation of the method).

5.3 Dimensions (in $\mathrm{mm}$ ) and geometry of an eighth of the tested specimen. . . . . . . .

5.4 Experimental displacement components across cross section $x=0$ containing the inclusion. (a): $u_{x} ;(\mathrm{b}): u_{y} ;(\mathrm{c}): u_{z} \ldots \ldots \ldots \ldots \ldots$

5.5 Experimental strain fields across cross section $x=0$ containing the inclusion. (a): $\varepsilon_{x x}$; (b): $\varepsilon_{y y} ;(\mathrm{c}): \varepsilon_{x x}+\varepsilon_{y y}+\varepsilon_{z z} \ldots \ldots \ldots \ldots$

5.6 Magnitude of the measured MR signal. . . . . . . . . . . . . . . . . . .

5.7 (a) Identified modulus distribution (dimensionless values) across all the available cross sections. (b) Reference modulus distribution (dimensionless values) across all the available cross sections. . . . . . . . . . . . . . . . . . . .

5.8 Identified modulus distribution across a given cross section for a simulated homogeneous solid subjected to uniaxial tension with white noise added to the input strain field. . . 36

5.9 Filtered identified modulus distribution (dimensionless values). . . . . . . . . . . . 37

5.10 Identified modulus distribution across all the available cross sections obtained by the strain imaging approach (dimensionless values) 


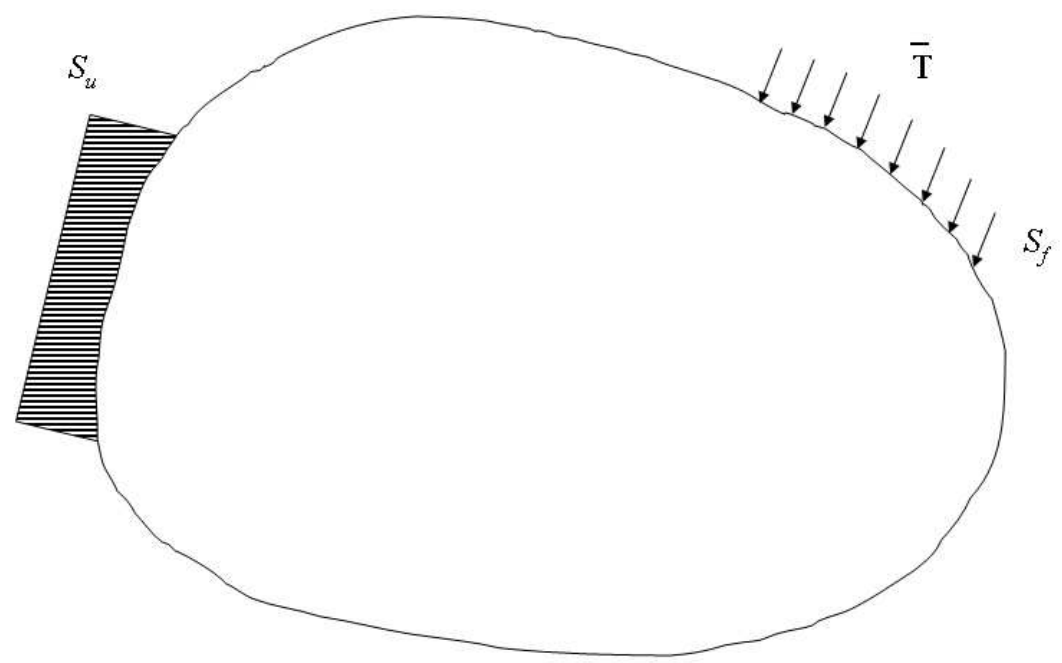

Fig. 5.1. Solid of any shape loaded on its boundary. 


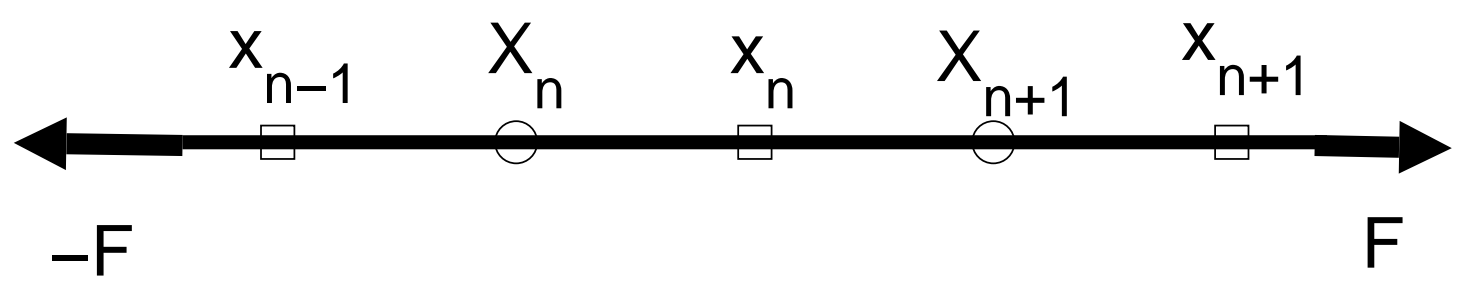

Fig. 5.2. 1D solid bar divided in spar finite elements (for the physical interpretation of the method). 


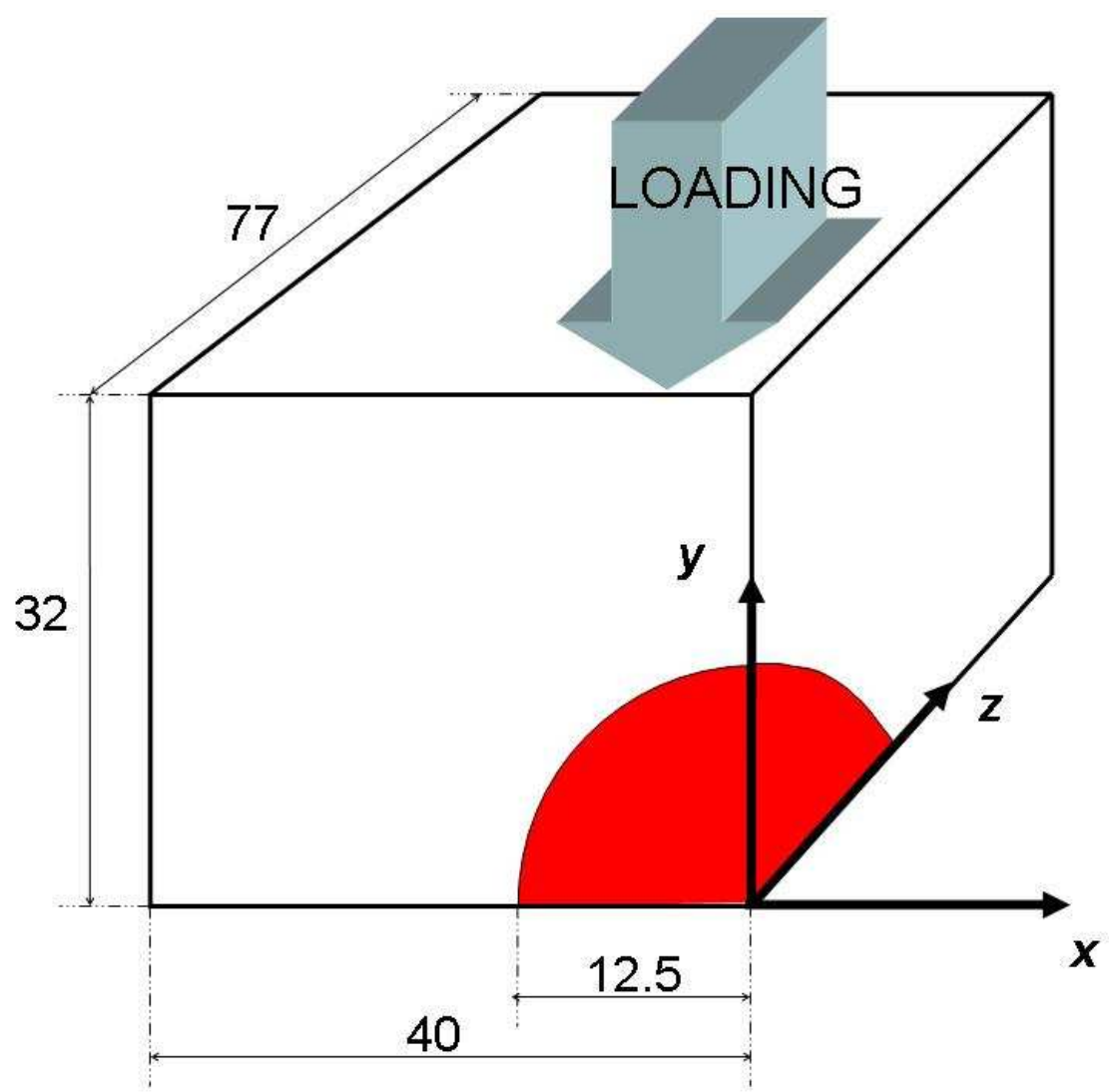

Fig. 5.3. Dimensions (in $\mathrm{mm}$ ) and geometry of an eighth of the tested specimen. 
(a)

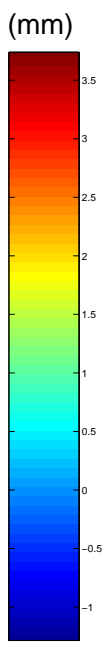

(b)

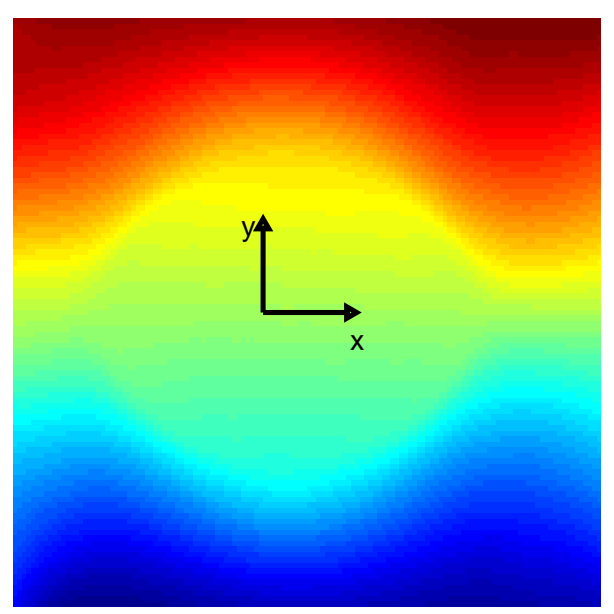

$(\mathrm{mm})$

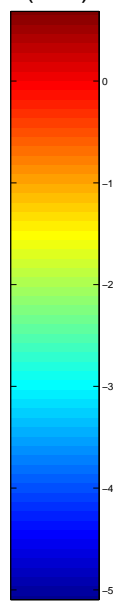

(c)

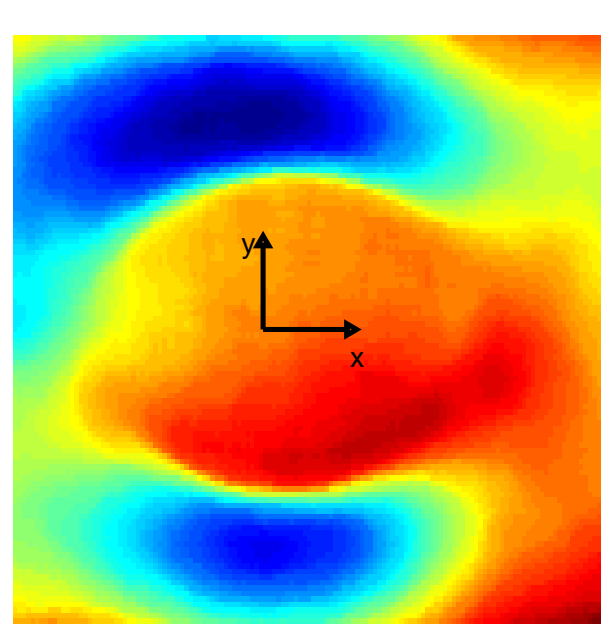

$(\mathrm{mm})$

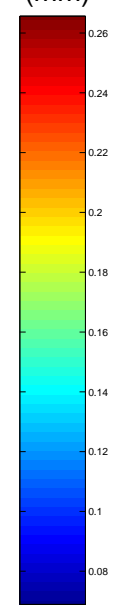

Fig. 5.4. Experimental displacement components across cross section $x=0$ containing the inclusion. (a): $u_{x} ;(\mathrm{b}): u_{y}$; (c): $u_{z}$. 
(a)

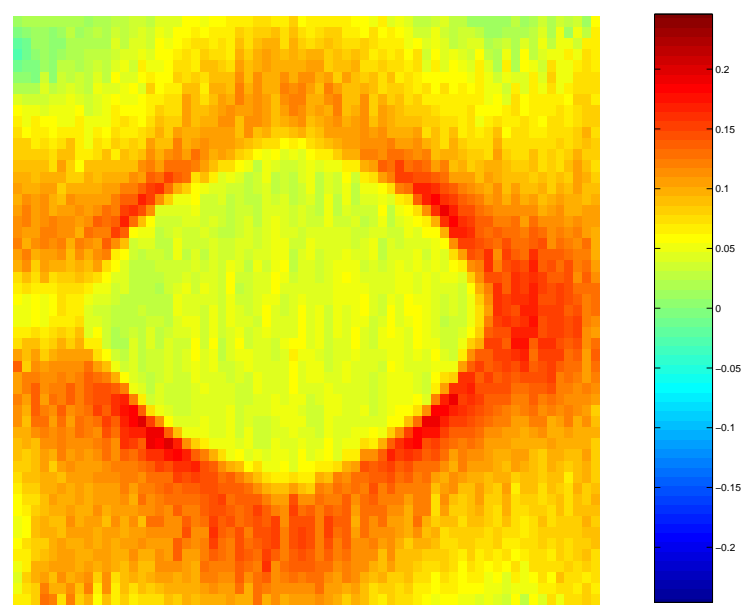

(b)

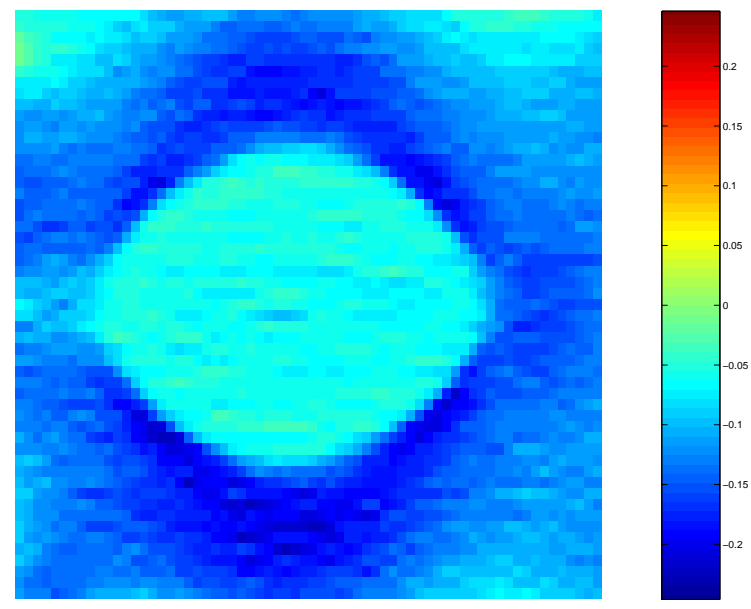

(c)
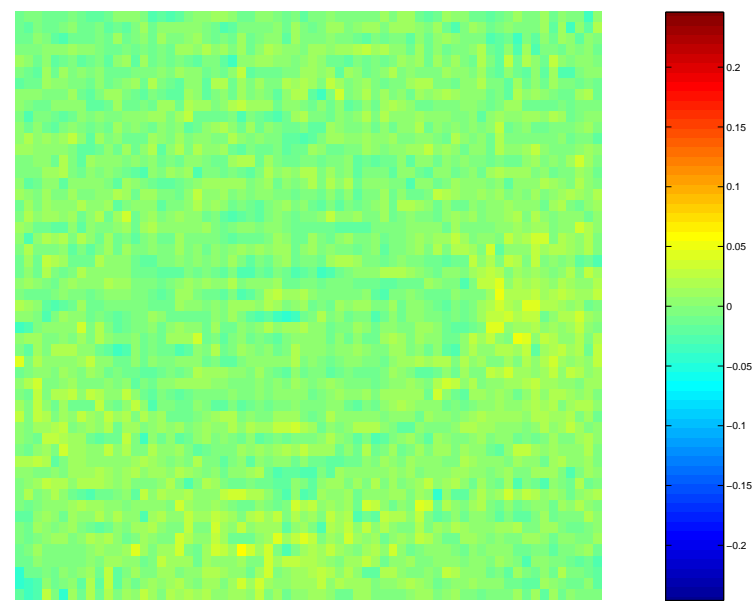

Fig. 5.5. Experimental strain fields across cross section $x=0$ containing the inclusion. (a): $\varepsilon_{x x} ;(\mathrm{b}): \varepsilon_{y y} ;(\mathrm{c}): \varepsilon_{x x}+$ $\varepsilon_{y y}+\varepsilon_{z z}$. 


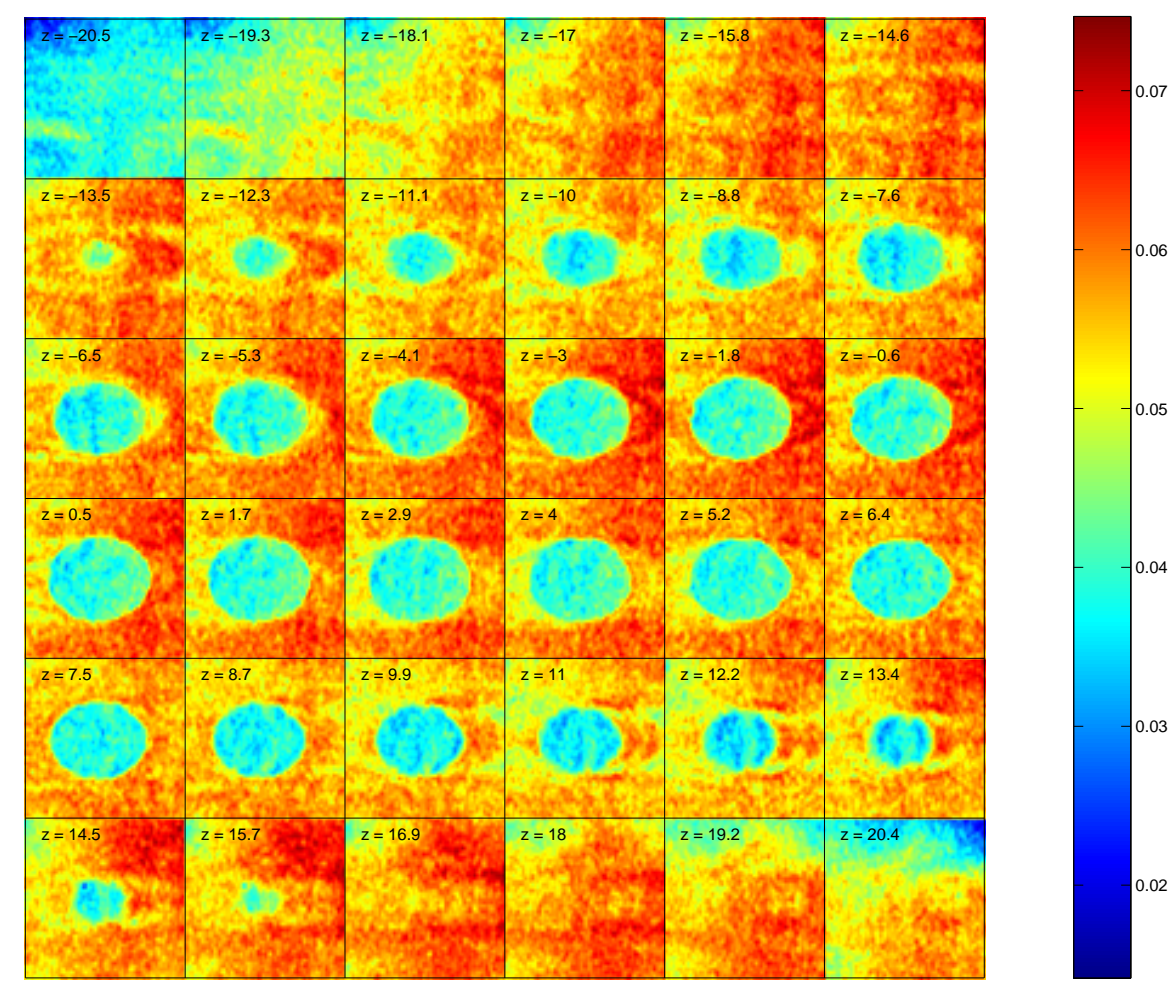


(a)
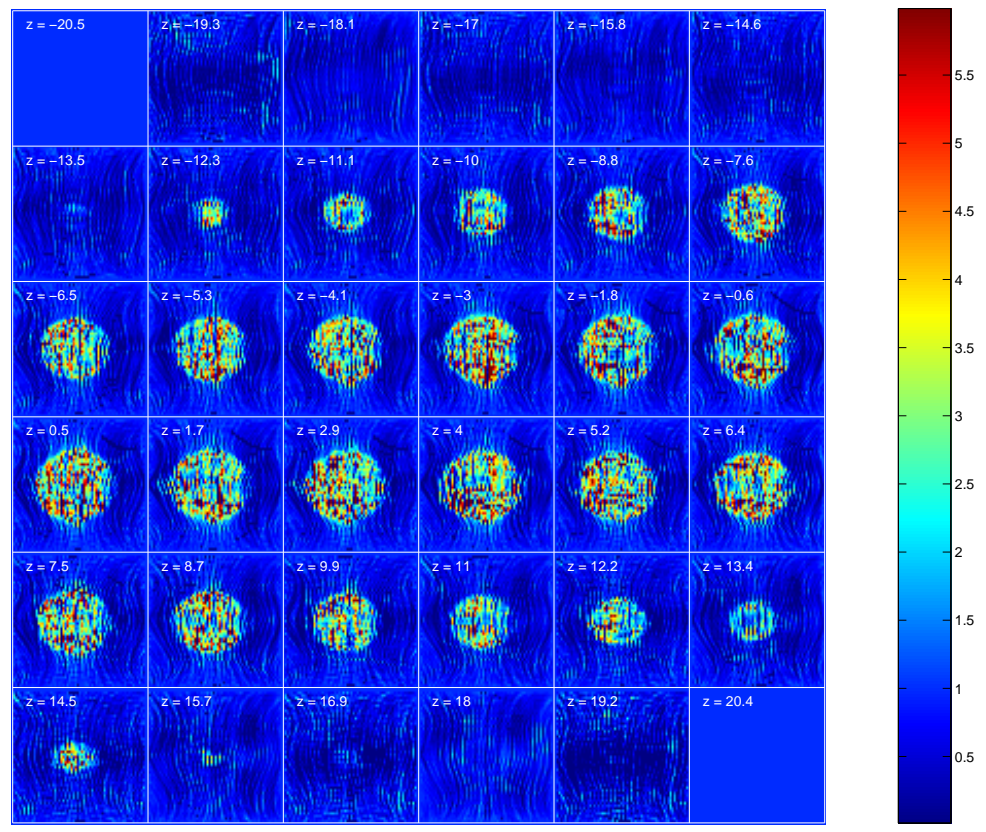

(b)
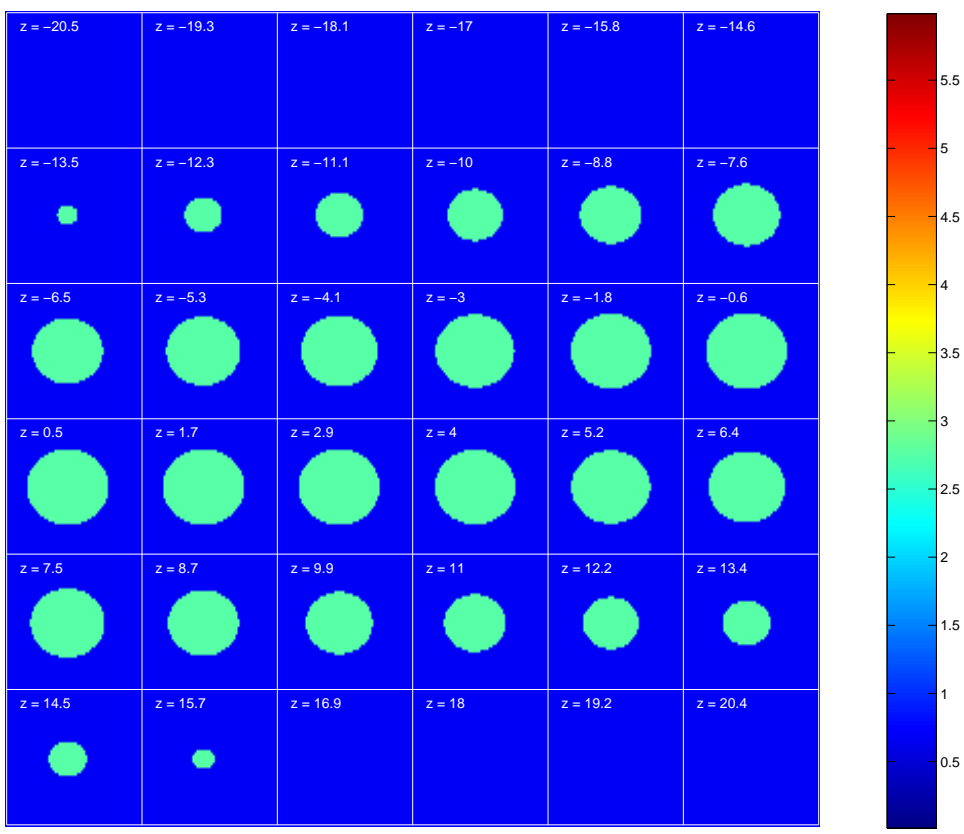

Fig. 5.7. (a) Identified modulus distribution (dimensionless values) across all the available cross sections. (b) Reference modulus distribution (dimensionless values) across all the available cross sections. 


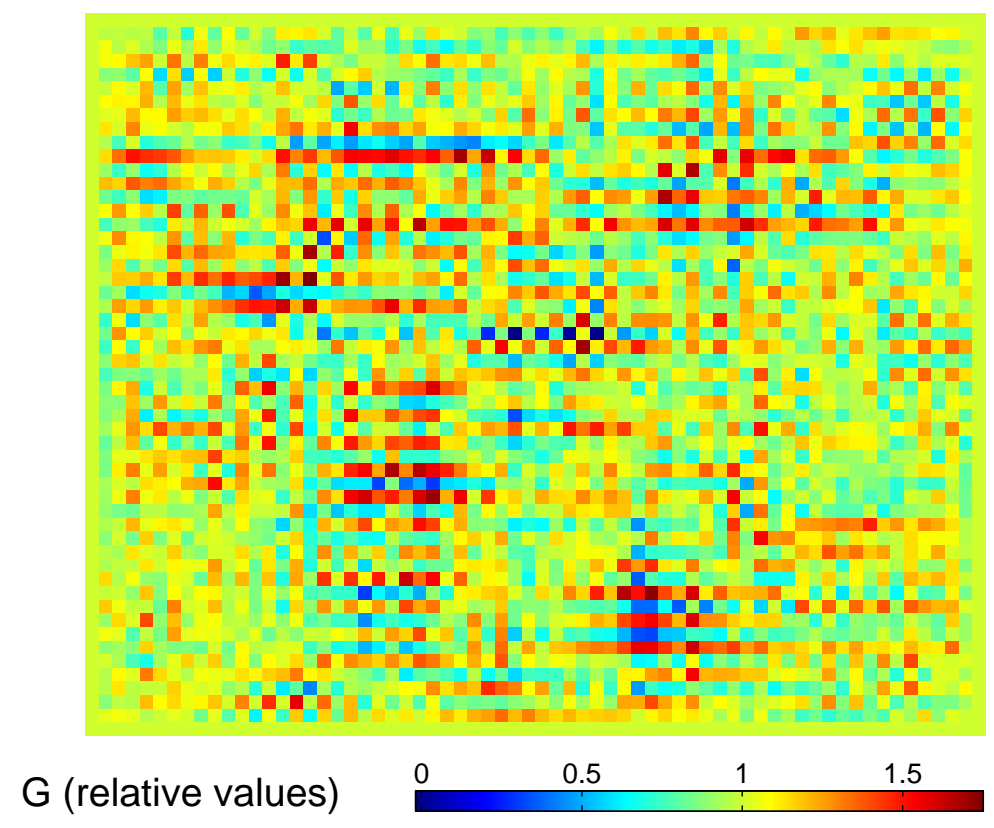

Fig. 5.8. Identified modulus distribution across a given cross section for a simulated homogeneous solid subjected to uniaxial tension with white noise added to the input strain field. 


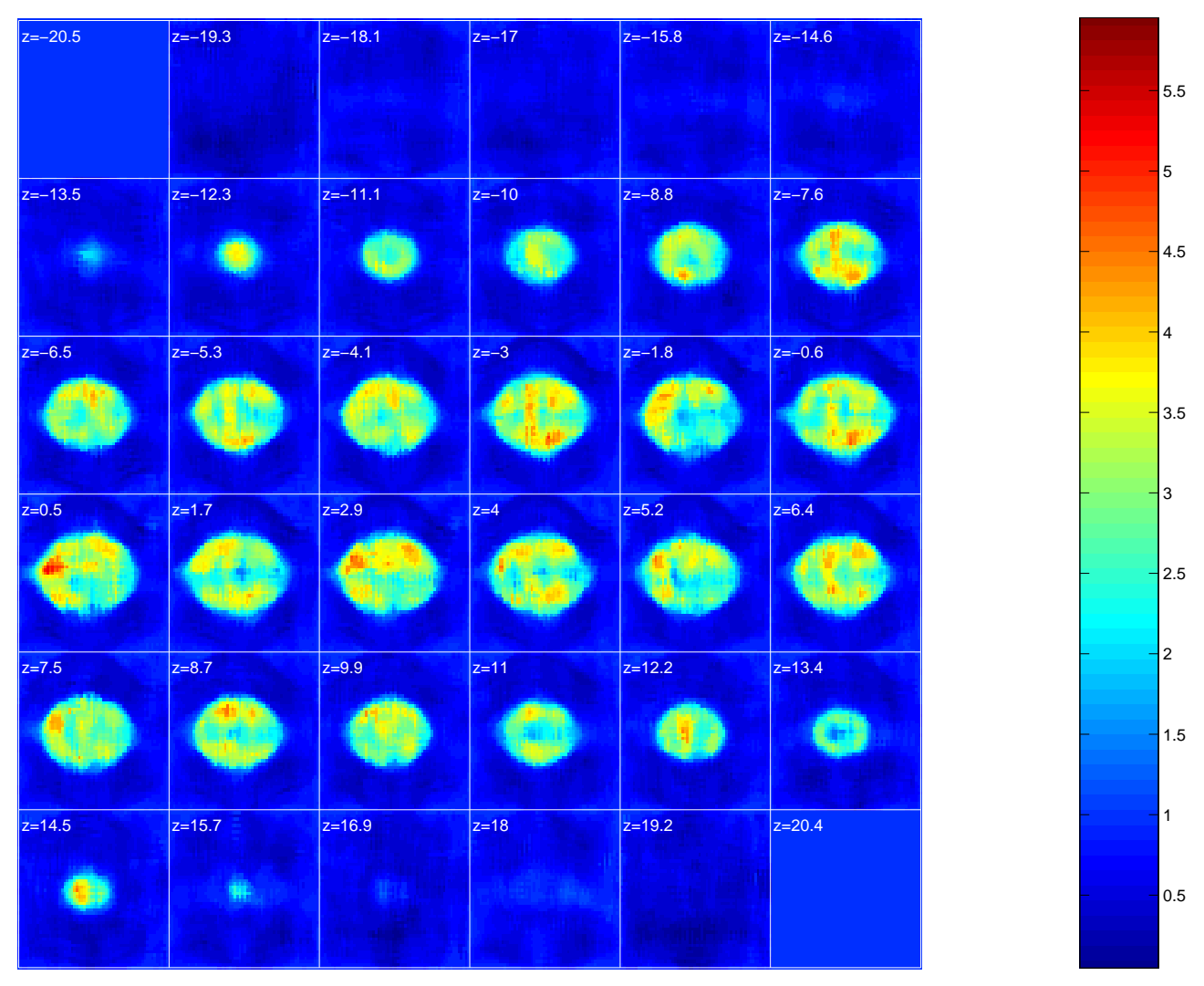




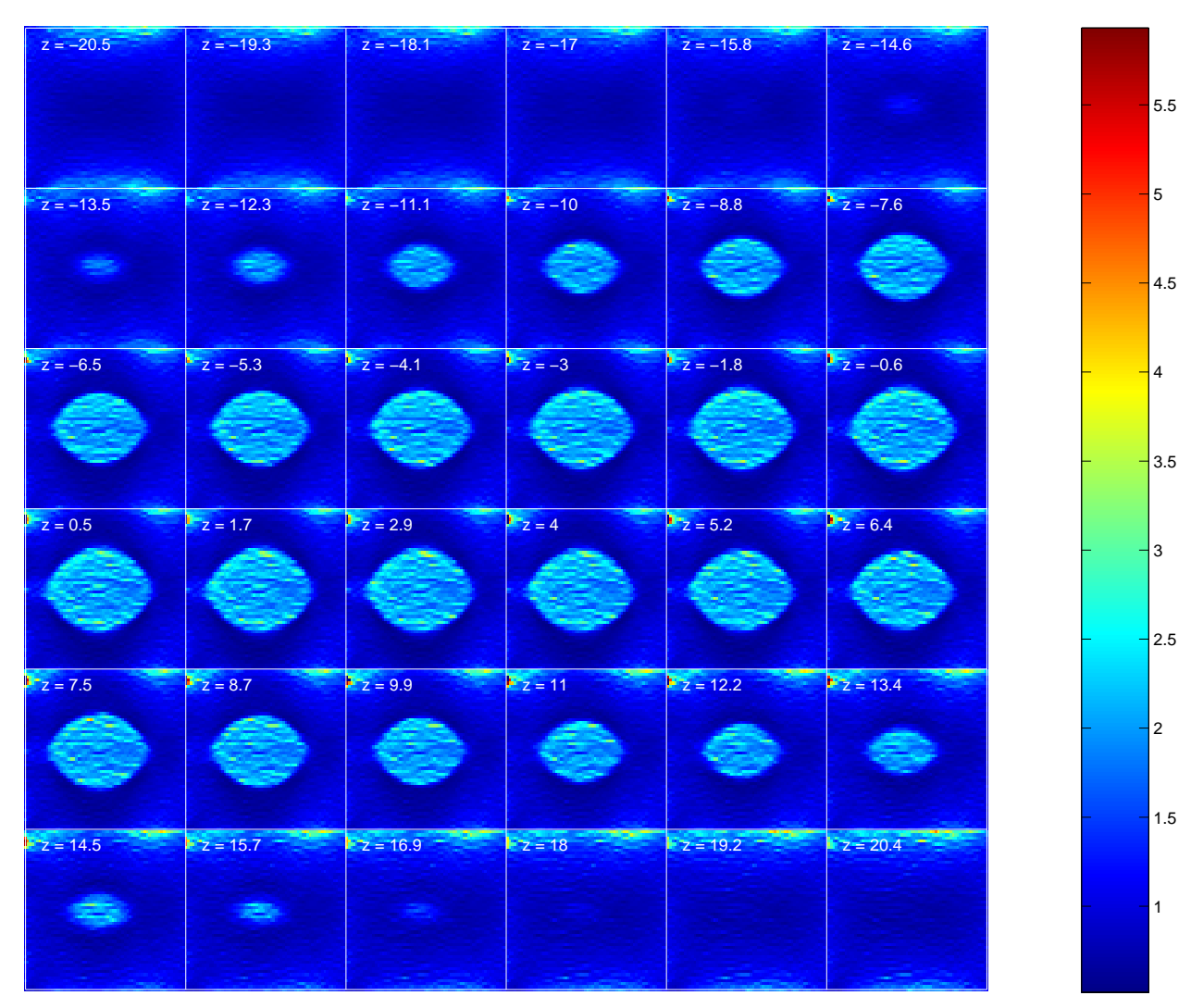

